OPEN ACCESS

Edited by:

Jun Zhang,

University of Kansas Medical Center,

United States

Reviewed by:

Keqiang Zhang,

City of Hope National Medical Center,

United States

Gianluca Baldanzi,

Università degli Studi del Piemonte

Orientale, Italy

*Correspondence: Conghua Xie chxie_65@whu.edu.cn Yan Gong

yan.gong@whu.edu.cn

Specialty section:

This article was submitted to

Thoracic Oncology,

a section of the journal

Frontiers in Oncology

Received: 29 October 2021 Accepted: 23 December 2021 Published: 17 January 2022

Citation:

Han L, Shi H, Ma S, Luo Y, Sun W,

Li S, Zhang N, Jiang $X$, Gao $Y$, Huang Z, Xie $C$ and Gong Y (2022) Agrin Promotes Non-Small Cell Lung

Cancer Progression and Stimulates Regulatory T Cells via Increasing IL-6 Secretion Through PI3KJAKT Pathway.

Front. Oncol. 11:804418. doi: 10.3389/fonc.2021.804418

\section{Agrin Promotes Non-Small Cell Lung Cancer Progression and Stimulates Regulatory T Cells via Increasing IL-6 Secretion Through PI3K/AKT Pathway}

\author{
Linzhi Han ${ }^{1}$, Hongjie Shi ${ }^{2}$, Shijing Ma ${ }^{1}$, Yuan Luo ${ }^{1}$, Wenjie Sun ${ }^{1}$, Shuying $\mathrm{Li}^{1}$, \\ Nannan Zhang ${ }^{1}$, Xueping Jiang ${ }^{1}$, Yanping Gao ${ }^{1}$, Zhengrong Huang ${ }^{3,4}$, \\ Conghua Xie ${ }^{1,5 *}$ and Yan Gong ${ }^{3,4 *}$
}

\begin{abstract}
' Department of Radiation and Medical Oncology, Zhongnan Hospital of Wuhan University, Wuhan, China, ${ }^{2}$ Department of Thoracic and Cardiovascular Surgery, Zhongnan Hospital of Wuhan University, Wuhan, China, ${ }^{3}$ Department of Biological Repositories, Zhongnan Hospital of Wuhan University, Wuhan, China, ${ }^{4}$ Tumor Precision Diagnosis and Treatment Technology and Translational Medicine, Hubei Engineering Research Center, Zhongnan Hospital of Wuhan University, Wuhan, China, ${ }^{5}$ Hubei Key Laboratory of Tumor Biological Behaviors, Hubei Cancer Clinical Study Center, Zhongnan Hospital of Wuhan University, Wuhan, China
\end{abstract}

Non-small cell lung cancer (NSCLC) has high mortality rates worldwide. Agrin contributes to immune synapse information and is involved in tumor metastasis. However, its roles in NSCLC and tumor immune microenvironment remain unclear. This study examined the effects and the underlying mechanisms of Agrin in NSCLC and tumor-infiltrated immune cells. Clinical tissue samples were used to confirm the bioinformatic predictions. NSCLC cells were used to investigate the effects of Agrin on cell cycle and proliferation, as well as invasion and migration. Tumor xenograft mouse model was used to confirm the effects of Agrin on NSCLC growth and tumor-infiltrated regulatory T cells (Tregs) in vivo. Agrin levels in NSCLC cells were closely related to tumor progression and metastasis, and its function was enriched in the PI3K/AKT pathway. In vitro assays demonstrated that Agrin knockdown suppressed NSCLC cell proliferation and metastasis, while PI3K/AKT activators reversed the inhibitory effects of Agrin deficiency on NSCLC cell behaviors. Agrin expression was negatively associated with immunotherapy responses in NSCLC patients. Agrin knockdown suppressed Tregs, as well as interleukin (IL)-6 expression and secretion, while PI3K/AKT activators and exogenous IL-6 rescued the inhibitory effects. In the mouse model, Agrin downregulation alleviated NSCLC cell growth and Treg infiltration in vivo. Our results indicated that Agrin promotes tumor cell growth and Treg infiltration via increasing IL-6 expression and secretion through PI3K/AKT pathway in NSCLC. Our studies suggested Agrin as a therapeutically potential target to increase the efficacy of immunotherapy in NSCLC patients.

Keywords: agrin, non-small cell lung cancer, regulatory T cell, interleukin-6, immunosuppression 


\section{INTRODUCTION}

Lung cancer has the leading occurrence (11.6\% of the total cases) and remains the most lethal one (18.4\% of the total deaths) all over the world (1). Non-small cell lung cancer (NSCLC) is the major histologic subtype, accounting for approximately $85 \%$ lung cancer (2). Despite the improvements in both traditional and novel treatments for NSCLC patients, only partial patients could benefit from these therapies, and the survival rates of the majority remain poor $(3,4)$.

Agrin is a glycosylated proteoglycan protein involved in the development of neuromuscular junctions (5). Recent studies show that Agrin plays important roles in cancers and immune system. Agrin was reported to be upregulated in various cancers than the adjacent normal tissues (6-9). Further studies found that Agrin upregulation enhanced tumorigenesis and metastasis via activating focal adhesion kinase and mitogen-activated protein kinase signaling pathways $(6,10)$. Moreover, Agrin was reported to activate $\mathrm{T}$ cells and facilitate immune connection formation between $\mathrm{T}$ cells and target cells $(11,12)$. Furthermore, Agrin was highly expressed on the membrane of primary $\mathrm{T}$ cells and involved in autoimmune disease progression (13). These results suggested that Agrin modulated cancer development and tumor immune microenvironment (TIME), which might be a potential target for tumor immunotherapy. Although Agrin was highly expressed in NSCLC and associated with worse survival, the specific roles of Agrin in NSCLC and TIME are still to be investigated (14).

In our study, we found that Agrin enhanced NSCLC development and regulated tumor-associated regulatory $\mathrm{T}$ cell (Treg) infiltration via enhancing interleukin-6 (IL-6) expression and secretion through PI3K/AKT pathway, suggesting that Agrin acted as an oncogene and augmented Tregs in NSCLC immune microenvironment. Our findings provided Agrin as a predictive factor of therapeutical approaches for NSCLC patients.

\section{MATERIALS AND METHODS}

\section{Data Collection and Preprocessing}

RNA-sequencing data (FPKM values) from 33 types of cancers were obtained from the Cancer Genome Atlas (TCGA; https:// portal.gdc.cancer.gov). Among them, 510 NSCLC and 59 adjacent normal tissue samples were used for differential expression analysis of Agrin, survival analysis and pathway enrichment analysis. According to Agrin expression levels, 510 NSCLC samples were divided into the high- and low-expression groups. Limma R package was used to analyze the differentially expressed genes, which were regarded as the genes co-expressed with Agrin. These genes were then imported into Metascape (http://metascape.org) for pathway enrichment analysis subsequently. Tumor Immune Estimation Resource (TIMER; cistrome.shinyapps.io/timer) was used to identify tumorinfiltrated immune cells (15). Tumor Immune Dysfunction and Exclusion (TIDE; tide.dfci.harva rd.edu) was used to predict tumor immune evasion, as well as immune checkpoint inhibitor responses $(16,17)$.

\section{Clinical Samples}

A total of 21 NSCLC samples and their paired normal lung tissues were obtained from Zhongnan Hospital of Wuhan University (Wuhan, China) from January 2018 to April 2020. None of these patients received chemotherapy or radiotherapy before surgery. Patients' clinical characteristics were shown in Table 1. The studies involving human participants were reviewed and approved by the Institutional Review Board at Zhongnan Hospital of Wuhan University. The patients/ participants provided their written informed consent to participate in this study. No potentially identifiable human images or data is presented in this study.

\section{Mice}

BALB/c nude mice (5-6 weeks) were purchased from the Vital River Laboratories (Beijing, China). NSCLC H1975 cells $\left(1 \times 10^{6}\right.$ cells per $100 \mu \mathrm{l}$ ) were injected subcutaneously into the right armpits of the mice. Human peripheral blood mononuclear cells (PBMCs, $5 \times 10^{6}$ cells per mouse) were injected subcutaneously into the same place of right armpits 7 days later. The sizes of tumors were measured with digital vernier calipers every 3 days. The volumes of tumors were calculated: volume $=$ (length $\times$ width $\left.^{2}\right) / 2$. Mice were sacrificed 45 days after cell inoculation, and tumor tissues were collected and measured for weight and volume. The animal study was reviewed and approved by the Institutional Animal Care and Use Committee at Zhongnan Hospital of Wuhan University.

\section{Cells}

Human NSCLC cell lines (H1299, H1975, H460, A549, PC9), Jurkat T and THP-1 cells were purchased from the Type Culture Collection of the Chinese Academy of Sciences (Shanghai, China). Human bronchial epithelium cell line (BEAS-2B) was purchased from Guangzhou Cellcook Biotech. NSCLC, Jurkat T and THP-1 cells were cultured in RPMI-1640 (Hyclone, USA), while BEAS-2B cells were cultured in DMEM (Gibco, USA), supplemented with $10 \%$ fetal bovine serum (FBS, Hyclone). Human PBMCs were collected from healthy donors with informed consent and ethical approval. Monocytes and lymphocytes were harvested using lymphocyte separation medium (Dakewe, China) according to manufacturer's recommendations.

\section{RNA Interference}

Agrin was downregulated with siRNA-1 (sense: 5'- GCCUGCA AAUCUCUAUCCATT -3'; antisense: 5'- UGGAUAGAGA UUUGCAGGCTT -3'), siRNA-2 (sense: 5'-CCUUUGUC GAGUACCUCAATT -3'; antisense: 5'-UUGAGGUACUCGA CAAAGGTT -3') using jetPRIME ${ }^{\circledR}$ transfection reagent (Polyplus-transfection SA, France) following the manufacturer's instructions. Lentiviral-mediated shRNAs (GenePharma, China) were used for stable Agrin downregulation, and puromycin (Cayman, USA) was used to select cells at a final concentration of $4 \mu \mathrm{g} / \mathrm{ml}$. 
TABLE 1 | Patient characteristics.

\begin{tabular}{|c|c|c|c|c|}
\hline Variable & Low Agrin expression $(n=10)$ & High Agrin expression $(n=11)$ & $\begin{array}{l}\text { Total } \\
(n=21)\end{array}$ & p-value* \\
\hline Gender & & & & 0.048 \\
\hline Age & & & & 0.08 \\
\hline$<65 y$ & 7 & 3 & 10 & \\
\hline$\geq 65 y$ & 3 & 8 & 11 & \\
\hline No & 8 & 3 & 11 & \\
\hline Stage & & & & 0.39 \\
\hline 1 & 5 & 8 & 13 & \\
\hline$\|$ & 1 & 2 & 3 & \\
\hline III & 3 & 1 & 4 & \\
\hline IV & 1 & 0 & 1 & \\
\hline$N$ & & & & 0.75 \\
\hline NO & 6 & 7 & 13 & \\
\hline N1 & 1 & 2 & 3 & \\
\hline N2 & 3 & 2 & 5 & \\
\hline M & & & & 0.9 \\
\hline MO & 9 & 11 & 18 & \\
\hline M1 & 1 & 0 & 1 & \\
\hline Lymph_node_status & & & & 0.88 \\
\hline metastasis & 3 & 4 & 7 & \\
\hline No-metastasis & 7 & 7 & 14 & \\
\hline Tumor size & & & & 0.66 \\
\hline$<3.5 \mathrm{~cm}$ & 7 & 6 & 13 & \\
\hline$\geq 3.5 \mathrm{~cm}$ & 3 & 5 & 8 & \\
\hline
\end{tabular}

The bold p-values indicate the statistically significant difference.

\section{Quantitative Real-Time PCR}

Total RNAs were obtained using TRIzol (Vazyme, China). RNA $(1 \mu \mathrm{g})$ was reversely transcribed to cDNA using the HiScript ${ }^{\circledR} \mathrm{Q}$ RT SuperMix kit (Vazyme). Quantitative PCR (qPCR) was performed using the ChamQTM SYBR ${ }^{\circledR}$ qPCR Master Mix (Vazyme) on the BIO-RAD CFX96. The relative expression levels of mRNAs were assessed by the $2-\Delta \Delta C$ t method. The primers are listed in Table S1.

\section{Immunoblotting}

Total proteins were extracted using RIPA cell lysis buffer (Beyotime, China) containing phosphatase and protease inhibitors (Sigma, USA). After centrifugated at $13,000 \mathrm{rpm}$ at $4^{\circ} \mathrm{C}$ for $10 \mathrm{~min}$, supernatants were obtained and boiled with $5 \times$ SDS loading buffer for $10 \mathrm{~min}$. Bicinchoninic acid system (Beyotime) was used to detect protein concentrations. The proteins were separated by $10 \%$ SDS-PAGE gels and transferred onto PVDF membranes (Merck). The membranes were blocked with 5\% non-fat milk for $90 \mathrm{~min}$, and then incubated with specific primary antibodies at $4^{\circ} \mathrm{C}$ overnight. The primary antibodies are presented in Table S2. After washing, the membranes were incubated with HRP-conjugated antibodies at room temperature for 2 hours. After washing, the proteins were exposed to ECL developer (Aspen) and analyzed by Bio-Rad Image Lab software.

\section{Immunofluorescence}

The cells on $24 \times 24 \mathrm{~mm}$ glass slides were fixed with $4 \%$ paraformaldehyde (PFA, Sangon, China) and permeabilized with $0.5 \%$ Triton X-100 (Beyotime) for $15 \mathrm{~min}$. After washing and blocking with $5 \%$ bovine serum albumin, the cells were incubated with anti-Ki67 antibodies (1:200) at $4^{\circ} \mathrm{C}$ overnight. Secondary antibodies (1:200) were applied for 1 hour. Nuclei were stained with DAPI (Sigma), and images were obtained by fluorescent microscope (Olympus, Japan).

\section{Colony Formation and Cell Proliferation Assays}

For colony formation assay, NSCLC cells were cultured in 6-well plates for 2 weeks. The cells were then fixed with $4 \%$ PFA, and stained with $0.5 \%$ crystal violet (Beyotime). After washing, the numbers of colonies were counted under microscope. For cell proliferation assay, NSCLC cells were seeded into 96-well plates. Cell viability was measured by the CCK- 8 reagent kit (Vazyme). The optical density values were estimated at $450 \mathrm{~nm}$ everyday using a multimodal plate reader (Molecular Devices, USA).

\section{Modified Boyden Chamber Assay}

NSCLC cells were suspended in RPMI-1640 with 1\% FBS and planted into the upper chamber, while RPMI-1640 with 10\% FBS was added to the lower chamber. After incubation for 24 hours, 
the filters were fixed with 4\% PFA, and then stained with $0.5 \%$ crystal violet. Cells in the upper surface of the chamber were wiped using a cotton swab. Cells at $\times 100$ magnification were counted and photographed. For the invasion assay, the upper chambers were pre-coated with Matrigel (BD, USA, dilution 1:40) before inoculation.

\section{Wound Healing Assay}

NSCLC cells were seeded into 6-well plates until full confluence. After scratched with a $1 \mathrm{ml}$ pipette tip, the migration rates were assessed: wound closure $(\%)=($ distance of initial scratched distance of final imaged without cells)/distance of initial scratched 48 hours later.

\section{Enzyme-Linked Immunosorbent Assay (ELISA)}

The supernatants were collected from H1299 and H1975 cells 48 hours after transfection. The levels of IL- 6 were measured by ELISA kit (Bioswamp, China). The optical density 450 values were determined using SpectraMax ${ }^{\circledR}$ Absorbance Reader.

\section{Flow Cytometry}

Single-cell suspensions of NSCLC cells or PBMCs were stained with the cell cycle staining kit (Beyotime), FITC anti-human CD4, APC anti-human CD25, or PE anti-human Foxp3 antibodies (Biolegend). The stained cells were washed and incubated with $1 \mathrm{ml}$ intracellular fixation buffer at room temperature for $60 \mathrm{~min}$. Permeabilization buffer $(2 \mathrm{ml})$ were then added into each tube, and the cells were centrifuged at 1,500 rpm for $7 \mathrm{~min}$. The supernatants were removed, and single-cell suspensions were analyzed by flow cytometry (Beckman, China).

\section{Immunohistochemistry (IHC)}

Tissue samples were fixed with 4\% PFA and embedded in paraffin. After cut into $4 \mu \mathrm{m}$ slides, deparaffinization, rehydration and blocking were performed in steps, followed by heat-induced antigen retrieval. Next, primary antibodies against Agrin (1:100, Zen), pAKT (1:100, Abclonal), Ki-67 (1:1000, Abcam), IL-6 (1:100, Abclonal), Foxp3 (1:100, Abcam) and CD8 (1:100, Abcam) were used to incubate the slides at $4^{\circ} \mathrm{C}$ for 12 hours. Consequently, corresponding secondary antibodies were used to incubate the slides at $37^{\circ} \mathrm{C}$ for 1 hour. Finally, the slides were stained with hematoxylin and then visualized on the light microscopy (Olympus, Japan).

\section{Statistical Analysis}

Data in this study were analyzed with Student's t-test and oneway ANOVA. All results were presented as Mean \pm standard error or mean \pm standard deviation (SD). $\mathrm{P}<0.05$ was defined as statistically significance.

\section{RESULTS}

\section{Agrin Is Upregulated in NSCLC and Associated With Worse Survival}

The landscape map demonstrated Agrin expression levels in 33 types of cancers (Figure 1A). As the most lethal tumor, lung cancer was selected to be further analyzed. Agrin was highly expressed in both lung adenocarcinoma and squamous cell carcinoma. The expression levels of Agrin were higher in the NSCLC tumor samples (510 cases) than the adjacent normal tissues (59 cases, $P<0.001$, Figure 1B). The patients with higher Agrin levels had lower long-term survival rates (Figure 1C). Moreover, 21 pairs of NSCLC and non-tumor lung tissues were collected from our hospital. Consistently, Agrin was significantly upregulated in tumors compared with the adjacent normal tissues (Figure 1D). The IHC results indicated that Agrin and pAKT expression were obviously higher in tumor than adjacent normal tissues (Figure 1E). Enrichment analysis also showed that Agrin was significantly associated with the PI3K/AKT pathway (Figure 1F).

\section{Agrin Downregulation Suppresses NSCLC Cell Proliferation and Induces Cell Cycle Arrest via Inhibiting PI3K/AKT Pathway}

Consistent with clinical samples, Agrin was also upregulated in NSCLC cells compared with regular lung epithelial cells (Figure S1A). H1299 and H1975 cells with high Agrin expression were transfected with specific siRNAs targeting Agrin, and the downregulation of Agrin was confirmed by PCR (Figures S1B, C). Immunoblotting results indicated that Agrin downregulation inhibited AKT phosphorylation, and that AKT activator SC79 restored this inhibition (Figures 2A, B). Colony formation assay found that Agrin deficiency decreased clonogenic capability of NSCLC cells, and that PI3K/AKT activators partially rescued this capability (Figures 2C, S2A, S4A). Moreover, Agrin knockdown suppressed NSCLC cell proliferation, while SC79 reversed these effects (Figures 2D, S2B). Immunofluorescence illustrated that Agrin downregulation decreased the numbers of Ki67 positive cells, and that SC79 partially restored the decrease (Figures 2E, S2C, E). Cell cycle assays showed that Agrin deficiency induced G0/G1 arrest, while SC79 reversed this arrest (Figures 2F, G, S2D, F). Immunoblotting results indicated that Agrin downregulation decreased CyclinD1 and CDK4/6 protein levels, and that PI3K/AKT activators rescued their expression (Figures 2H, S2G, S4B). These results suggested that Agrin deficiency suppressed NSCLC cell proliferation and induced cell cycle arrest via inhibiting PI3K/ AKT pathway.

\section{Agrin Silencing Inhibits NSCLC Cell Migration and Invasion via Suppressing PI3K/AKT Pathway}

In addition to cell proliferation, Agrin also regulated NSCLC cell metastasis. Modified Boyden chamber assay indicated that Agrin downregulation inhibited NSCLC cell migration and invasion, and that SC79 restored this inhibition (Figures 3A-C, S3C-F). Wound healing assay also suggested that depletion of Agrin significantly suppressed cell migration in both H1975 and H1299 cells, and that PI3K/AKT activators rescued this suppression (Figures 3D, S3A, B, S4C). In addition, lower levels of $\mathrm{N}$ Cadherin, Vimentin, MMP9, and higher levels of E-Cadherin were detected by immunoblotting in the Agrin silencing group (Figure S3G). However, SC79 and 740Y-P reversed these effects (Figures 3E, S4D). These results suggested that Agrin deficiency suppressed NSCLC cell metastasis through PI3K/AKT pathway. 
A

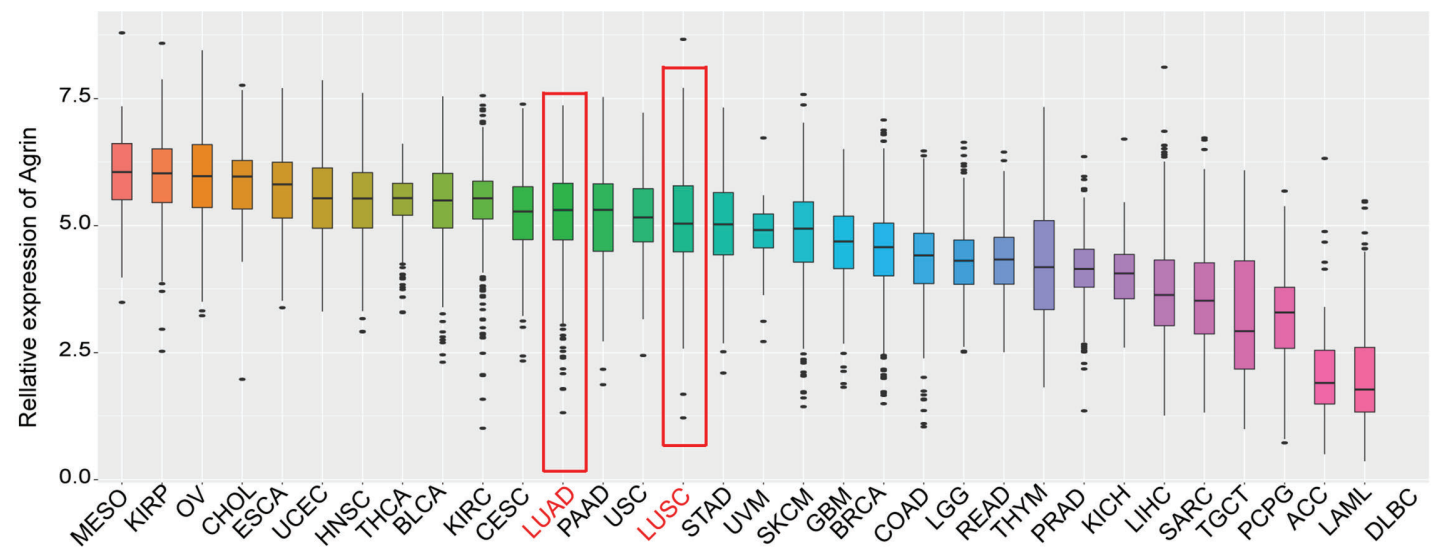

B

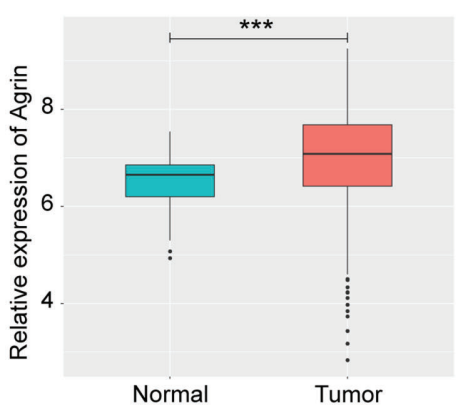

C

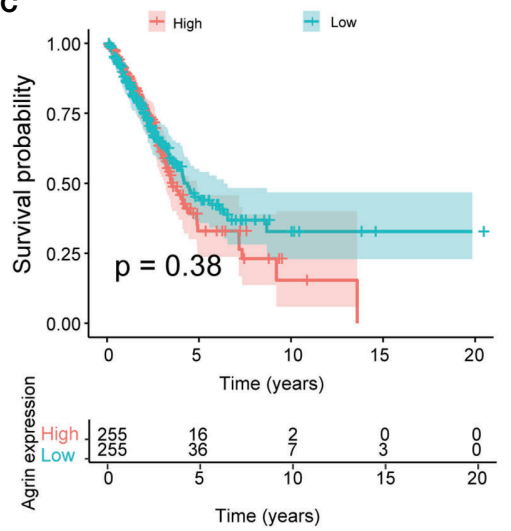

D

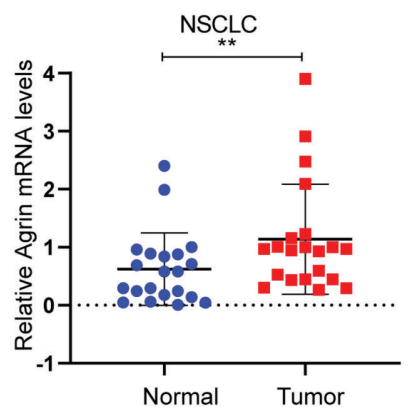

F
E

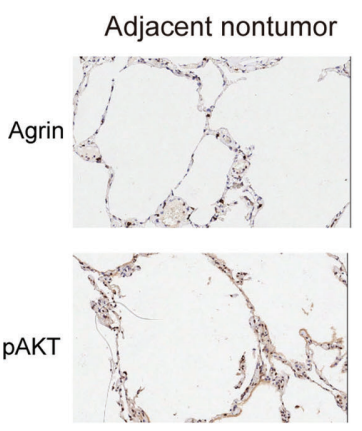

Tumor

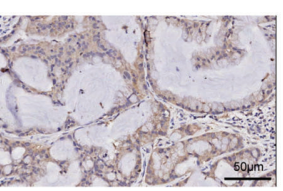

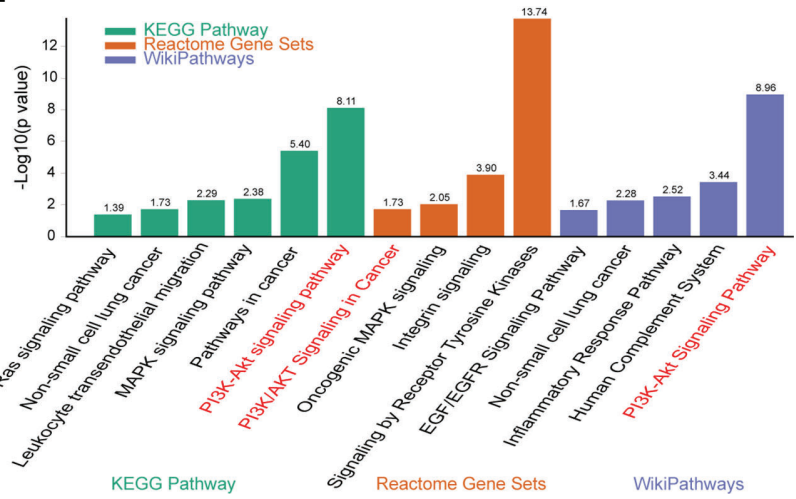

FIGURE 1 | Agrin was upregulated in NSCLC and associated with worse survival. (A) Agrin expression landscape in 33 types of cancer. (B) Differential expression of Agrin in NSCLC and adjacent normal samples. (C) Survival analysis for Agrin in NSCLC patients. (D) Differential expression of Agrin in 21 pairs of NSCLC and adjacent tissues. Agrin was significantly upregulated in NSCLC patients. (E) Representative IHC images of Agrin and pAKT staining in the clinical tumor and adjacent normal tissues. (F) Enrichment analysis of 3 databases (KEGG Pathway, Reactome Gene Sets, Wiki Pathways). ${ }^{* *} \mathrm{P}<0.01$; ${ }^{\star \star \star} \mathrm{P}<0.001$. Student's t-test.

\section{Agrin Deficiency Suppresses Tregs via Downregulating PI3K/AKT/IL-6 Signaling Pathway}

As Agrin was reported to be expressed in T cells and promote autoimmune disease development, we further explored the effects of Agrin on TIME using TIMER and TIDE website. The expression levels of Agrin were significantly associated with Treg infiltration and the response rates of immune checkpoint inhibitors in NSCLC patients (Figures 4A, B, 5A). After coculturing with NSCLC cells for 6 days, the Treg proportions in PBMCs were significantly increased, while the percentages of Tregs were less in PBMCs cocultured with Agrin-deficient NSCLC cells (Figures 4C, D). The Treg-related genes (TGFB1, Foxp3, CTLA-4, IL-10, PRF1, GZMB) were declined in Jurkat T 


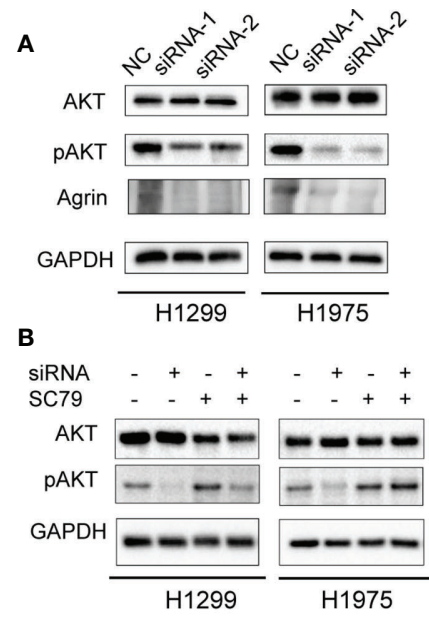

C
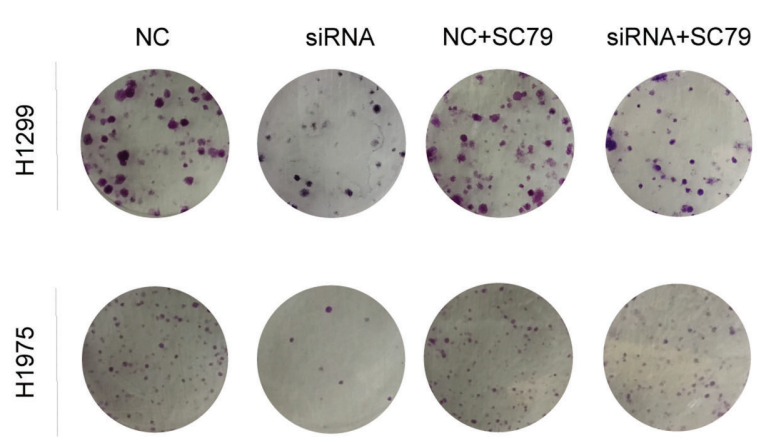

E
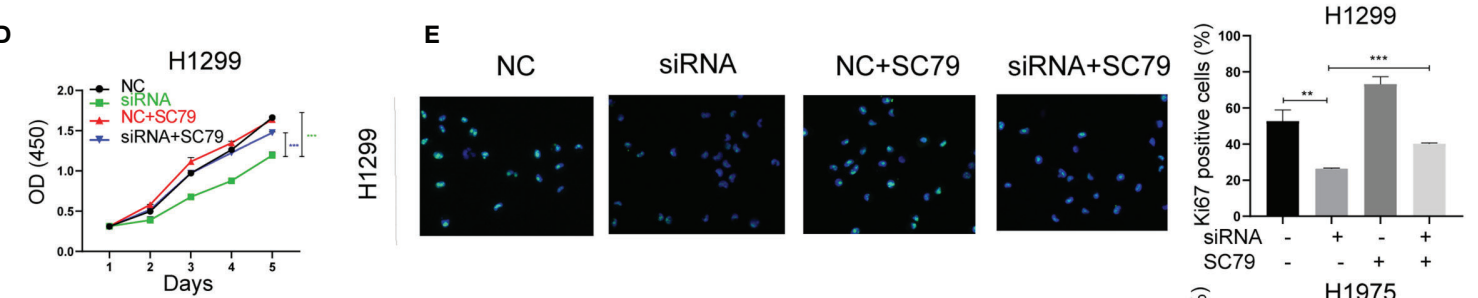

H1975
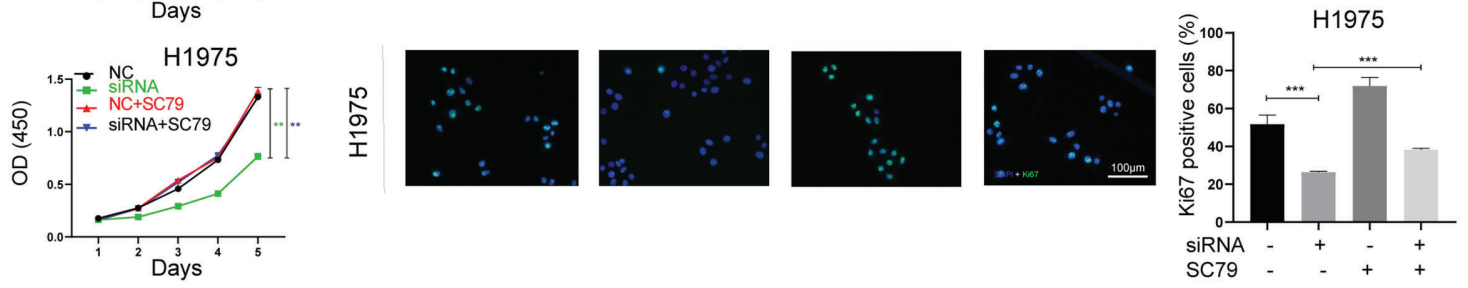

$\mathbf{F}$
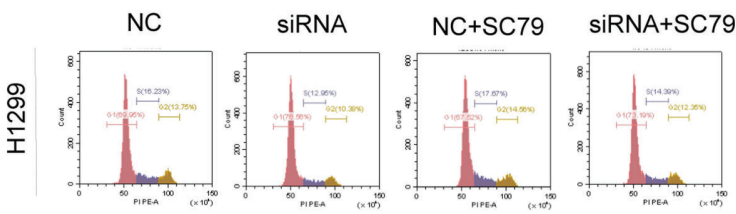

G
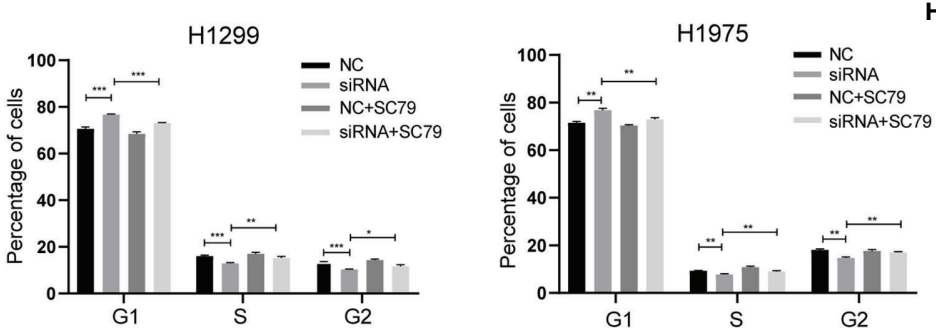

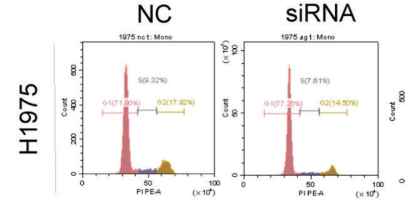

$\mathrm{NC}+\mathrm{SC} 79$ siRNA+SC79

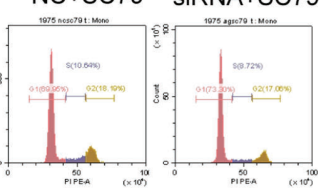

SC79 -+++

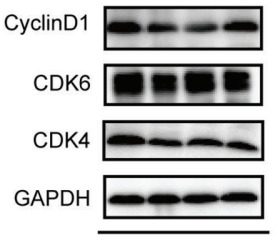

H1299

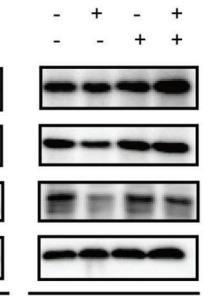

$\mathrm{H} 1975$

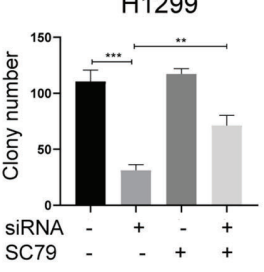

H1975

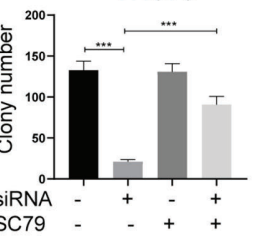

FIGURE 2 | Agrin downregulation suppressed NSCLC cell proliferation and induced cell cycle arrest via inhibiting PI3K/AKT pathway. (A, B) Agrin knockdown inhibited AKT phosphorylation in $\mathrm{H} 1299$ and $\mathrm{H} 1975$ cells. (C) SC79 $(5 \mu \mathrm{g} / \mathrm{ml})$ rescued colony formation inhibited by Agrin deficiency in NSCLC cells. (D) OD values of CCK-8 assay in NSCLC cells treated with Agrin siRNA and SC79. (E) Representative images of Ki-67 staining in NSCLC cells treated with Agrin siRNA and SC79. The images were taken at 400X magnification. Scale bar, $100 \mu \mathrm{m}$. (F, G) SC79 reversed G1 arrest induced by Agrin deficiency in NSCLC cells. (H) Representative immunoblots of CyclinD1 and CDK4/6. $N=3$; ${ }^{*} P<0.05 ;{ }^{* *} P<0.01 ;{ }^{* * *} P<0.001$.

cells and PBMCs cocultured with Agrin-deficient NSCLC cells (Figures 4E, F).Although M1 macrophages and CD8 T cells also played an important role in TIME, no significant alteration of macrophage polarization or CD8 $\mathrm{T}$ cells was detected in the THP-1 cells or PBMCs cocultured with Agrin-deficient NSCLC cells (Figures S5B, C). Moreover, treatment with SC79 or 740Y$\mathrm{P}$ in NSCLC cells could restore the proportions of Tregs in PBMCs and the Treg-related genes (Figures 5, S4F). There results revealed that Agrin silencing in NSCLC cells downregulated Tregs through PI3K/AKT signaling pathway. 
A
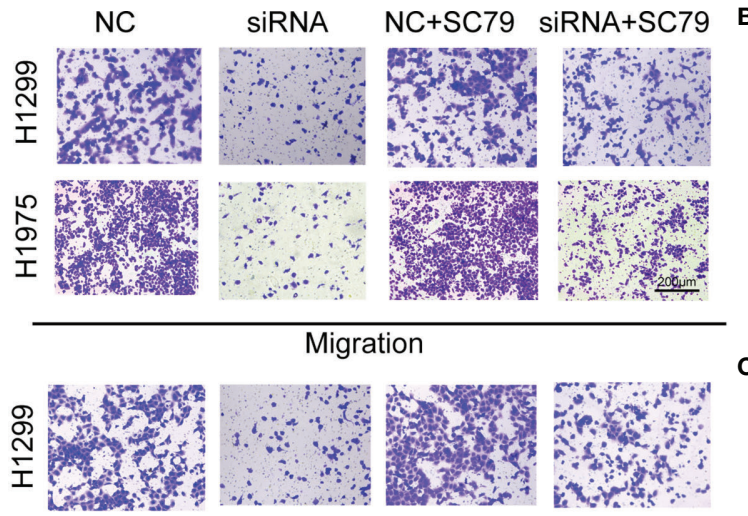

Migration
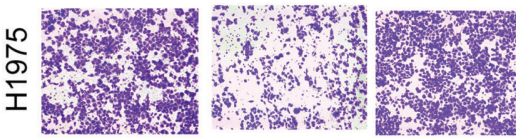

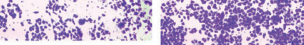

Invasion

D

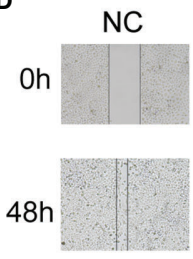
SiRNA
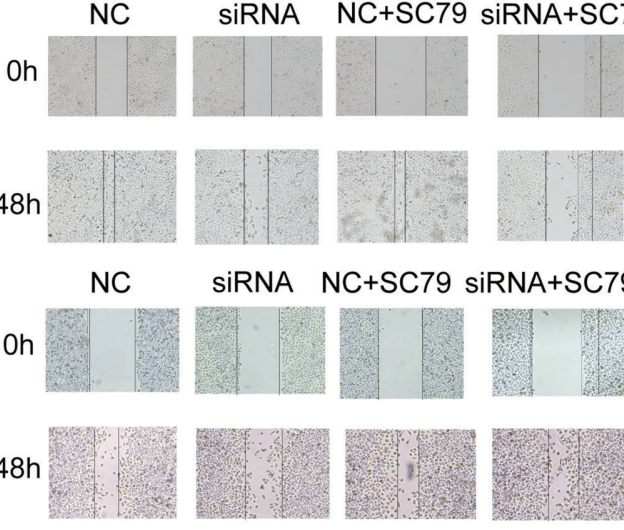

siRNA NC+SC79 siRNA+SC79
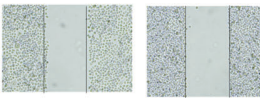

$48 \mathrm{~h}$
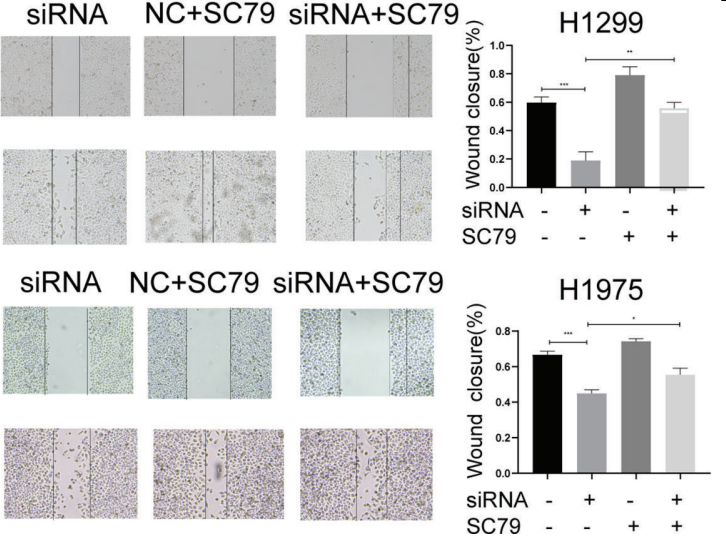
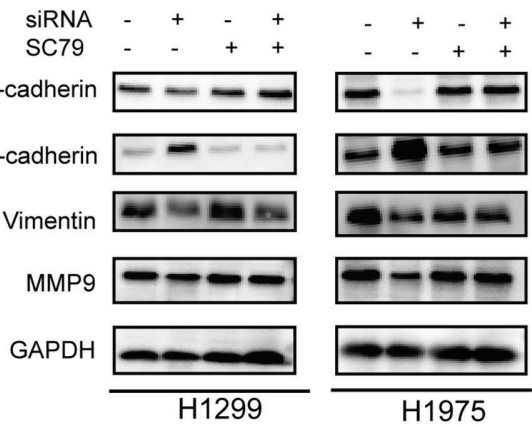

$\mathrm{H} 1975$

FIGURE 3 | Agrin silencing inhibited NSCLC cell migration and invasion via suppressing PI3K/AKT pathway. (A-C) Representative images of modified Boyden chamber migration and invasion assays in NSCLC cells treated with Agrin siRNA and SC79. Scale bar, $200 \mu \mathrm{m}$. (D) Representative images of wound healing assays in NSCLC cells treated with Agrin siRNA and SC79. The images were taken at 100X magnification. Scale bar, $200 \mu \mathrm{m}$. (E) Representative immunoblots of NCadherin, E-Cadherin, Vimentin and MMP9. $\mathrm{N}=3$; ${ }^{*} \mathrm{P}<0.05 ;{ }^{* \star} \mathrm{P}<0.01$; ${ }^{* \star} \mathrm{P}<0.001$

Several tumor-secreting cytokines, including IL-6, IL-10 and TGF-b1, are essential for Treg differentiation. To identify the mediators between NSCLC cells and Tregs, qPCR was used to screen these potential key cytokines in both H1299 and H1975 cells (Figure 6A). The results revealed that IL-6, rather than IL10 and TGF-b1, was significantly downregulated in the Agrindeficient NSCLC cells. ELISA confirmed the lower levels of IL-6 in the medium of Agrin-deficient NSCLC cells (Figure 6B). Moreover, the addition of PI3K/AKT activators reversed the decrease of IL- 6 mRNA levels in NSCLC cells and protein levels in the medium (Figures 6C, D). In addition, exogenous IL-6 treatment restored Treg proportions in PBMCs cocultured with Agrin-deficient NSCLC cells, while the addition of IL-6 antibodies significantly decreased the percentages of Tregs in PBMCs (Figures 6E-G). The results of immunoblotting indicated that Agrin silencing downregulated IL-6 in NSCLC cells, and that SC79 or 740Y-P could reverse these effects (Figures 6H, S4E). TIMER website revealed that IL-6 and Foxp3 were closely associated in NSCLC cells (Figure 6I). Collectively, these observations indicated that Agrin knockdown inhibited Treg differentiation via downregulating PI3K/AKT/IL-6 pathway.

\section{Agrin Knockdown Alleviates NSCLC Cell Growth and Treg Infiltration In Vivo}

To investigate the effects of Agrin downregulation on NSCLC cell growth and Treg infiltration in vivo, Agrin was downregulated with lentiviral-mediated shRNAs. After puromycin selection, both the transcriptional and protein levels of Agrin were significantly decreased (Figure S1D). In the xenograft tumor model using $\mathrm{BALB} / \mathrm{C}$ nude mice, Agrin deficiency significantly suppressed NSCLC cell growth in vivo (Figures $\mathbf{7 A - C}$ ). In the group of 

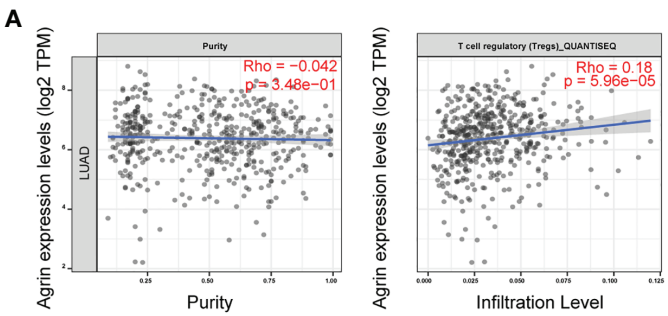

B
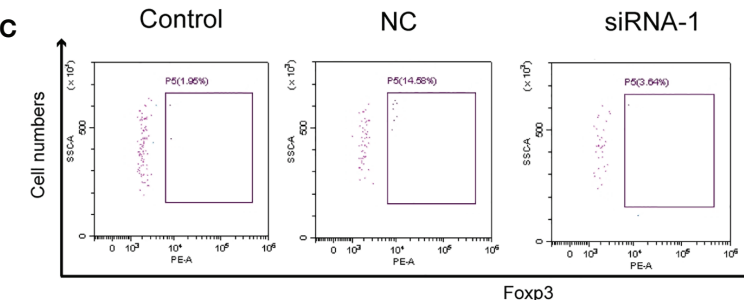

SiRNA-1
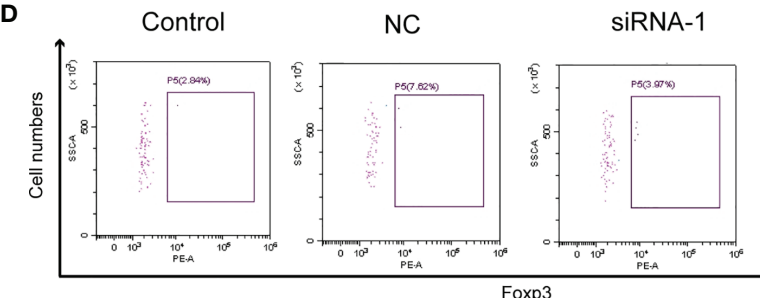
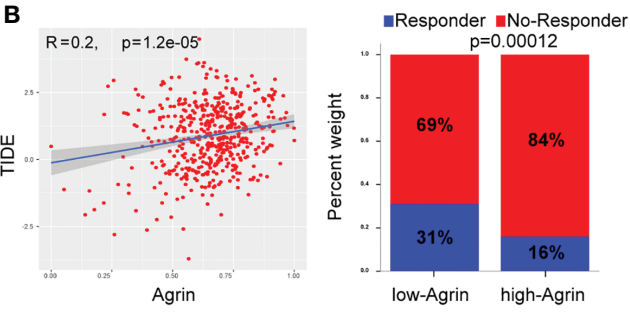

SiRNA-2
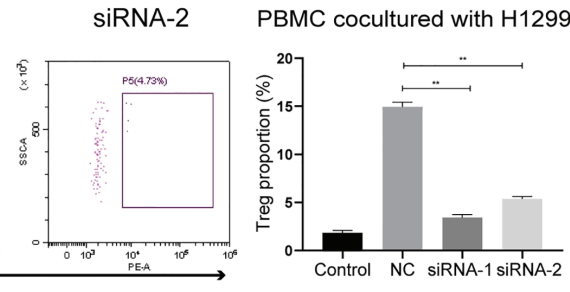

SiRNA-2

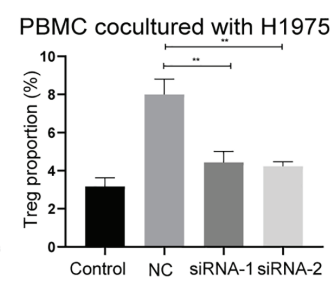

E
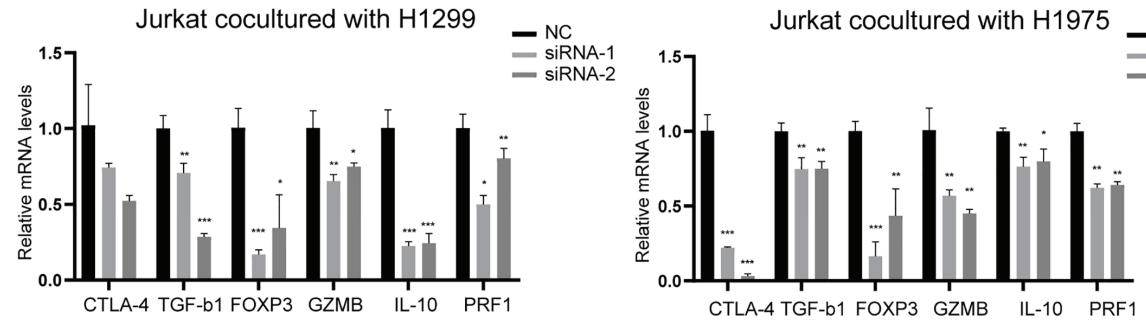

$\mathbf{F}$

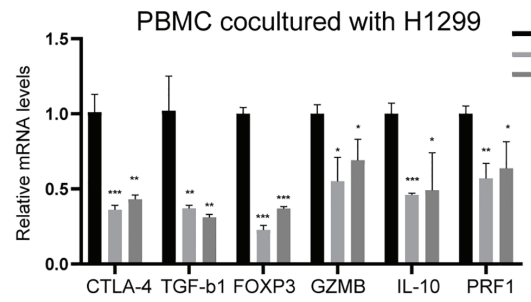

PBMC cocultured with $\mathrm{H} 1975$

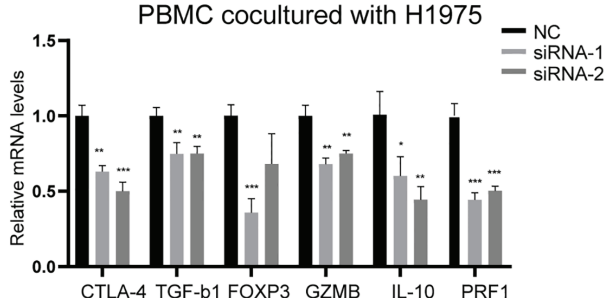

FIGURE 4 | Agrin knockdown decreased Treg differentiation and downregulated Treg-related genes. (A) The relationship between Treg infiltration and Agrin expression in NSCLC from TIMER. (B) The immunotherapeutic response rates for NSCLC patients in TIDE between the low- and high-Agrin groups. (C, D) Flow cytometry of Tregs (CD4+, CD25+, Foxp3+) in PBMCs cocultured with NSCLC cells transfected with NC or siRNA. (E, F) Relative expression of Treg markers (CTLA-4, TGF-b1, Foxp3, GZMB, IL-10, PRF1) in Jurkat T cells and PBMCs cocultured with NSCLC cells transfected with NC or siRNA for 6 days. $N=3$; ${ }^{\star} P<0.05 ;{ }^{\star *} P<0.01 ;{ }^{\star \star \star} P<0.001$.

PBMC injection, Agrin knockdown also inhibited tumor growth (Figures 7D-F). The IHC results indicated that Agrin knockdown inhibited NSCLC cell proliferation, IL-6 and p-AKT expression in vivo (Figure 7G). Moreover, Agrin deficiency decreased the expression levels of Foxp3 and IL- 6 in NSCLC TIME (Figure $7 \mathbf{H}$ ). Taken together, our results suggested that Agrin downregulation significantly suppressed NSCLC cell growth and Treg infiltration in vivo.

\section{DISCUSSION}

NSCLC is the most lethal malignancy worldwide. Due to its asymptomatic and rapid progression, most patients were diagnosed at advanced stages and had poor prognosis. Immunotherapy is a highprofile therapy by largely extending patients' survival time based on enriched immune-infiltrated environment in NSCLC (18-21). However, a considerable number of patients could not benefit from 
A
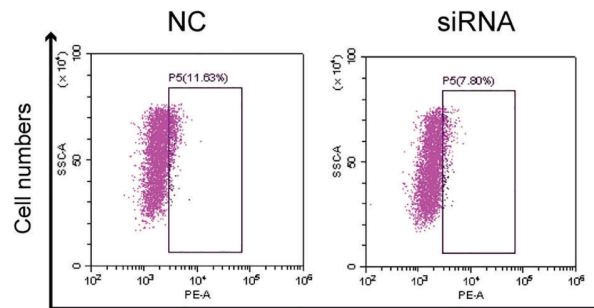

B

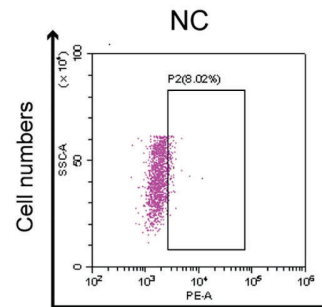

C

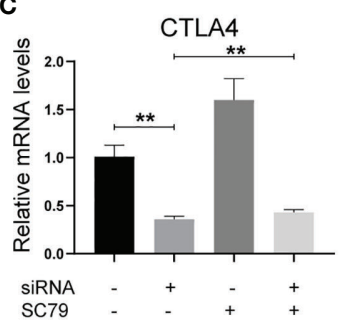

FOXP3
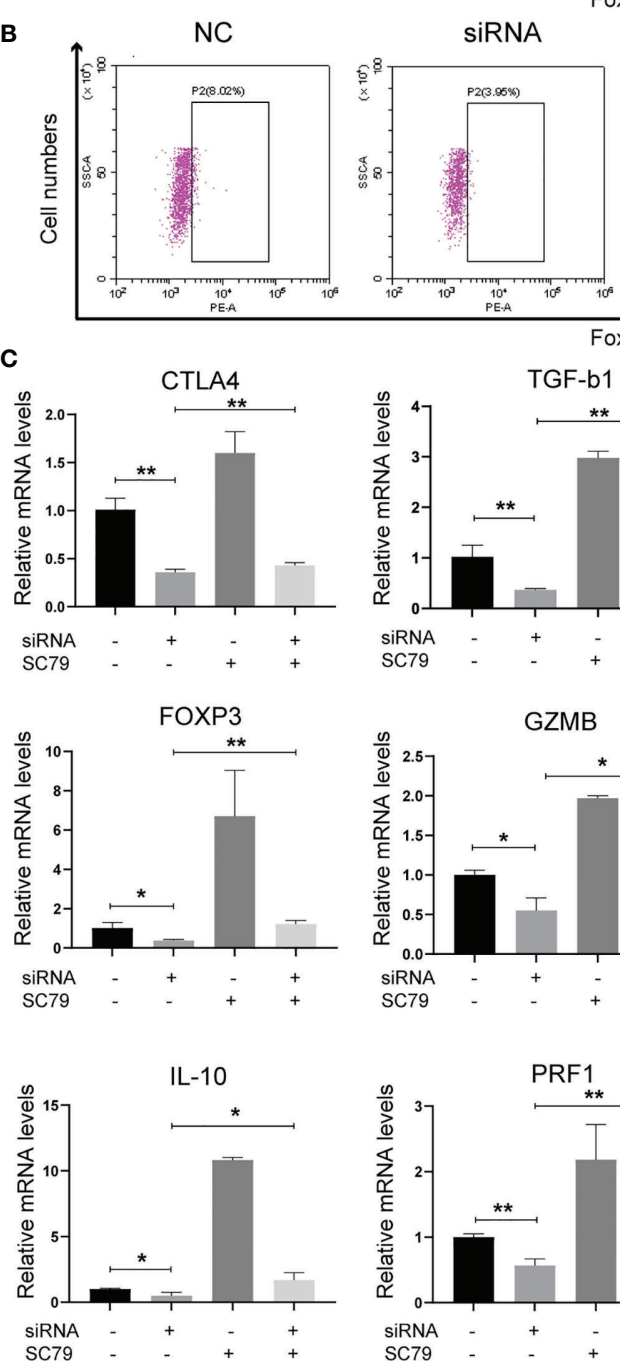

GZMB
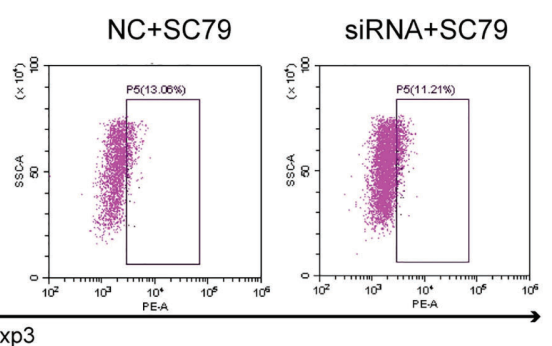

$\mathrm{NC}+\mathrm{SC} 79$

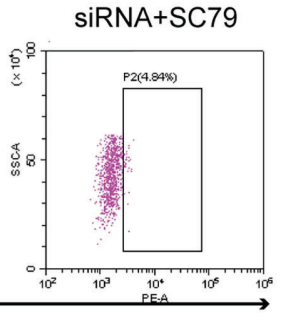

D
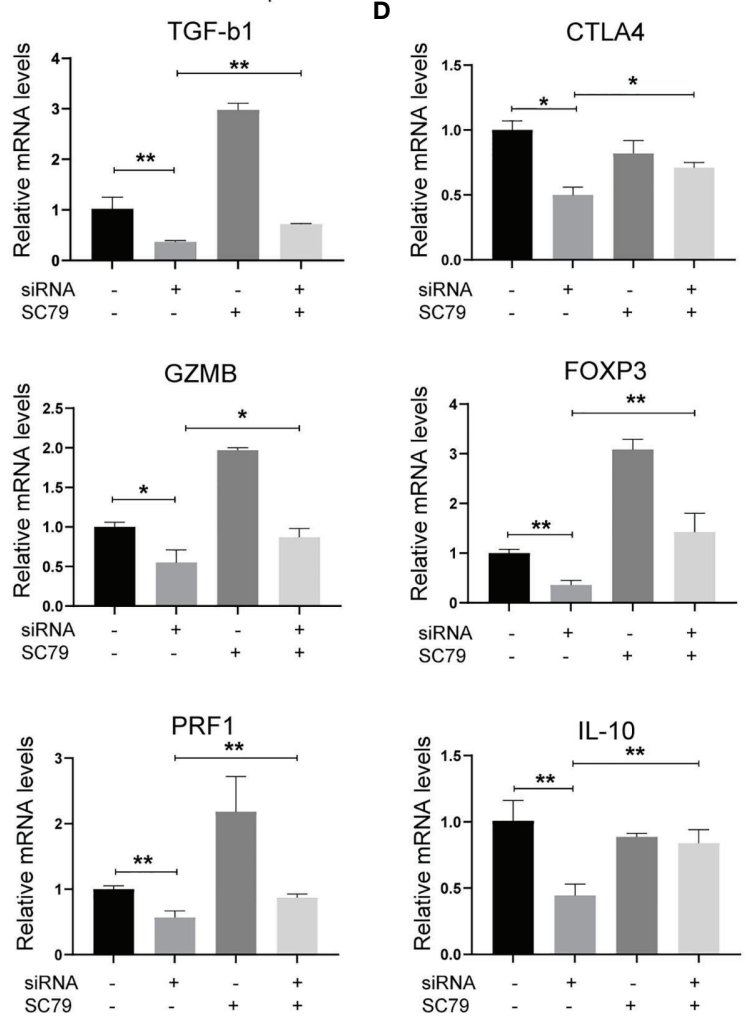

PBMC cocultured with $\mathrm{H} 1299$

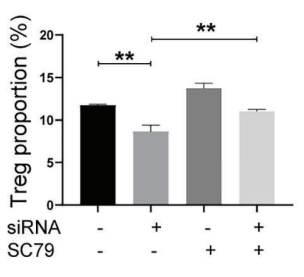

PBMC cocultured with $\mathrm{H} 1975$

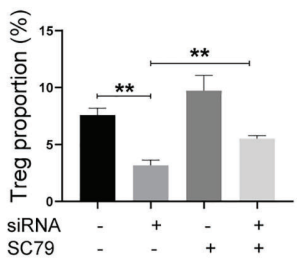

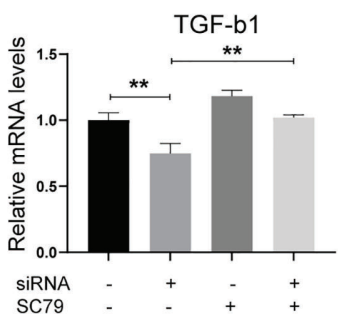
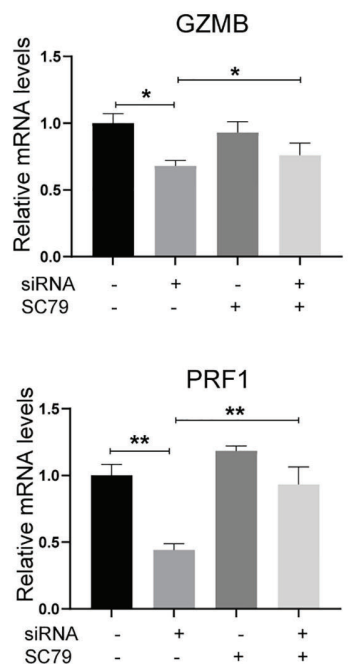

FIGURE 5 | Agrin regulated Treg differentiation and Treg-related genes expression through PI3K/AKT pathway. (A, B) Flow cytometry of Tregs (CD4+, CD25+, Foxp3+) in PBMCs cocultured with NSCLC cells treated with Agrin siRNA and SC79. (C, D) Relative expression of Treg markers (CTLA-4, TGF-b1, Foxp3, GZMB, IL-10, PRF1) in Jurkat T cells and PBMCs cocultured NSCLC cells treated with Agrin siRNA and SC79 for 6 days. $\mathrm{N}=3$; ${ }^{*} \mathrm{P}<0.05$; ${ }^{\star *} \mathrm{P}<0.01$.

immunotherapy. Recent studies illustrated that immune suppressive environment in NSCLC might hinder patients from getting profits from immune checkpoint inhibitors (22). Tumor progression has an inextricable association with TIME (23). Cancer cells not only enhance several oncogenic signaling pathways to promote tumor progression, but also secrete cytokines to stimulate and activate immunesuppressive cells, such as Tregs, tumor-associated fibroblasts and cancer stem cells, which in turn assist tumor to evade immune surveillance (24-29). Tregs repress tumor killing $\mathrm{T}$ cell responses (30), as the main impediments of cancer immunotherapy. Clinical researches approved that Treg exhaustion led to tumor regression (31) and induced anti-cancer immunity (32). Therefore, finding a way to inhibit tumor development and depleting Tregs might be effective to improve NSCLC prognosis. 
A

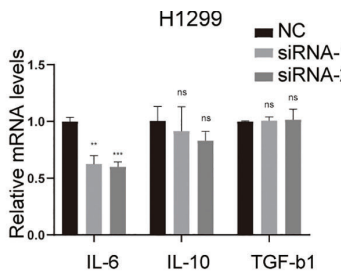

C

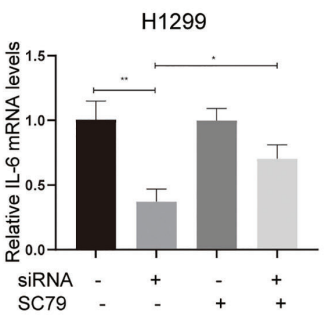

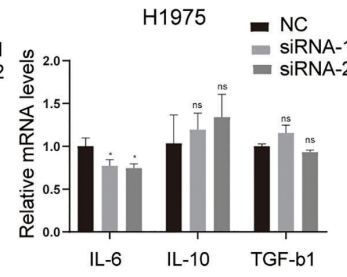
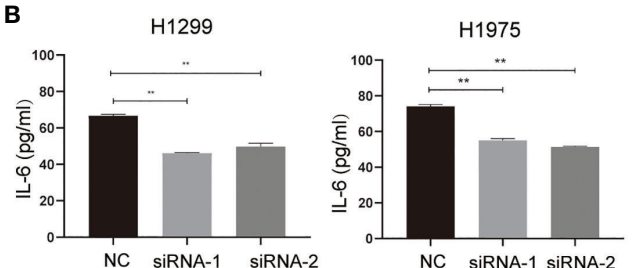

D
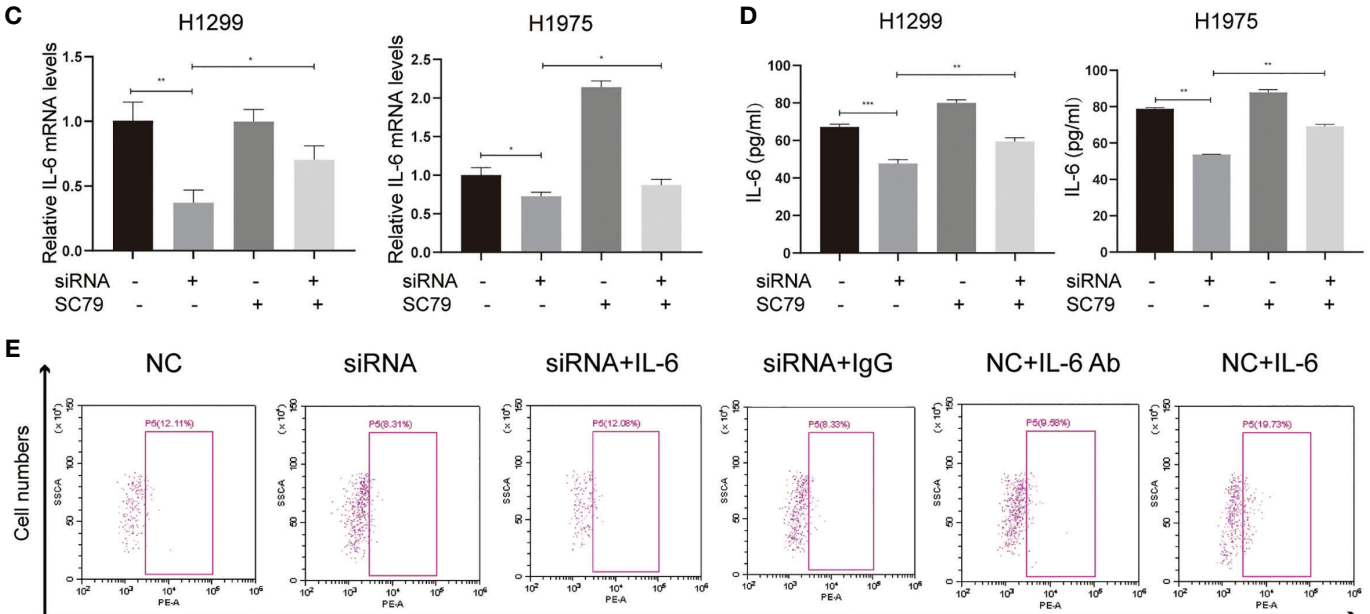

SiRNA+IL-6

siRNA+lgG
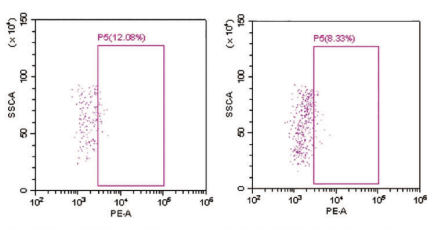

$N C+I L-6 A b$

NC+IL-6
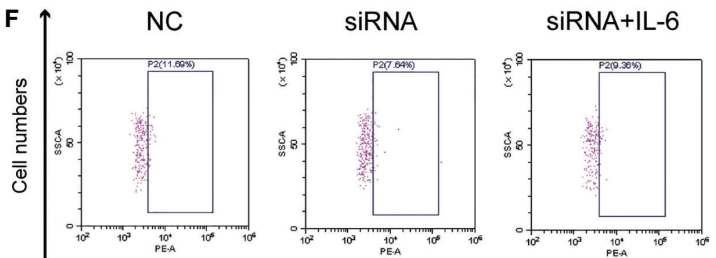

Foxp3
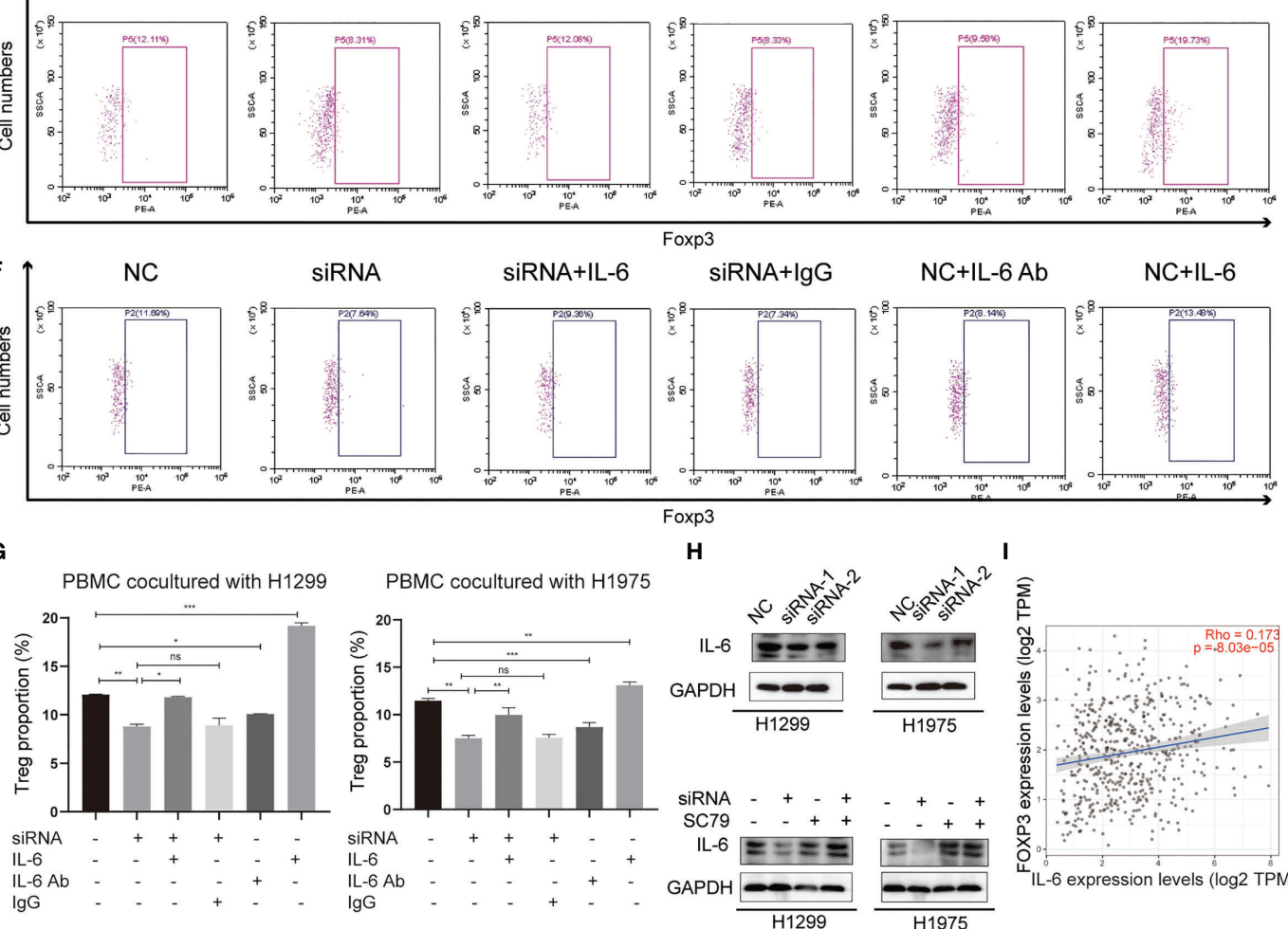

siRNA+lgG
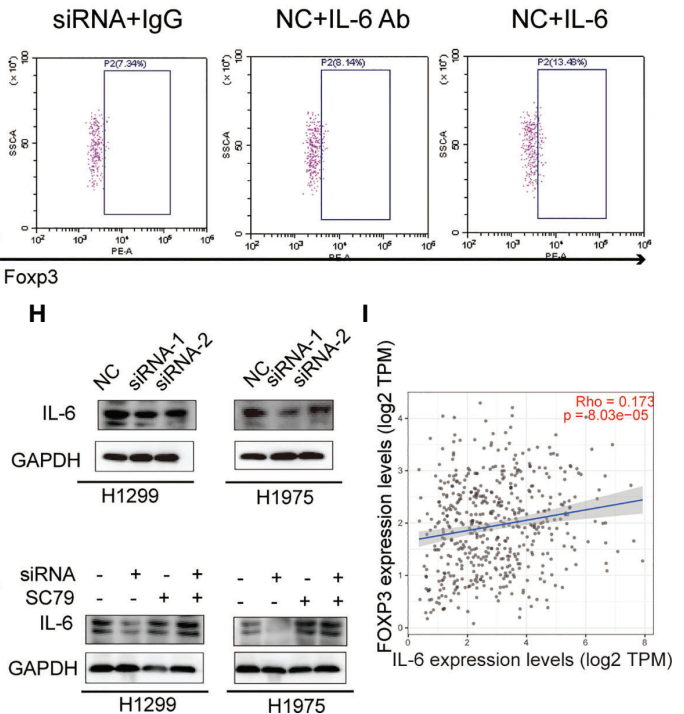

FIGURE 6 | Agrin regulated Treg differentiation via modulating IL-6 expression and secretion. (A) Relative mRNA levels of IL-6, IL-10 and TGF-b1 in NSCLC cells transfected with NC or Agrin siRNA. (B) ELISA of IL-6 secretion levels in NSCLC cells transfected with NC or siRNA. (C) Relative expression of IL-6 in NSCLC cells treated with Agrin siRNA and SC79. (D) ELISA of IL-6 secretion levels in NSCLC cells treated with Agrin siRNA and SC79. (E-G) Flow cytometry of Tregs (CD4+, CD25+, Foxp3+) in PBMCs cocultured with NSCLC cells treated with Agrin siRNA and IL-6/L-6 Ab. (H) Representative immunoblots of IL-6 in NSCLC cells treated with Agrin siRNA and SC79. (I) Correlation of IL-6 and Foxp3 expression in NSCLC (TIMER). N = 3; ns, nonsignificant; ${ }^{*} P<0.05 ;{ }^{* *} P<0.01$; ${ }^{* \star} P<0.001$.

Agrin exerts its vital roles in neuro synapse formation (5), autoimmune disease (13), angiogenesis (33) and various cancer development (6-9). In addition, Agrin is a key mediator in primary $\mathrm{T}$ cell activation in autoimmune disease (11-13). However, few study associated Agrin with TIME. In our study, samples from TCGA dataset suggested that Agrin was highly expressed in NSCLC compared with normal lung tissues and associated with worse survival. Data of qRT-PCR confirmed upregulated Agrin levels in NSCLC compared with the adjacent normal lung tissues. Subsequent experiments indicated that Agrin deficiency inhibited NSCLC cell proliferation and invasion. PI3K/ AKT pathway, a classical oncogenic signaling pathway, could 


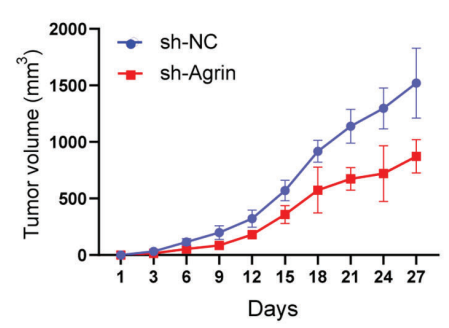

D

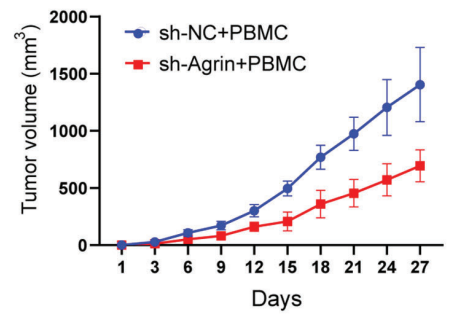

G

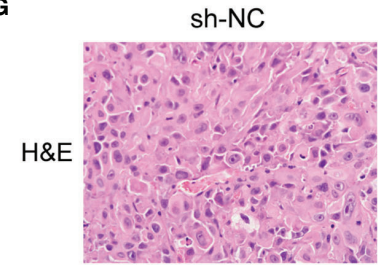

Ki67
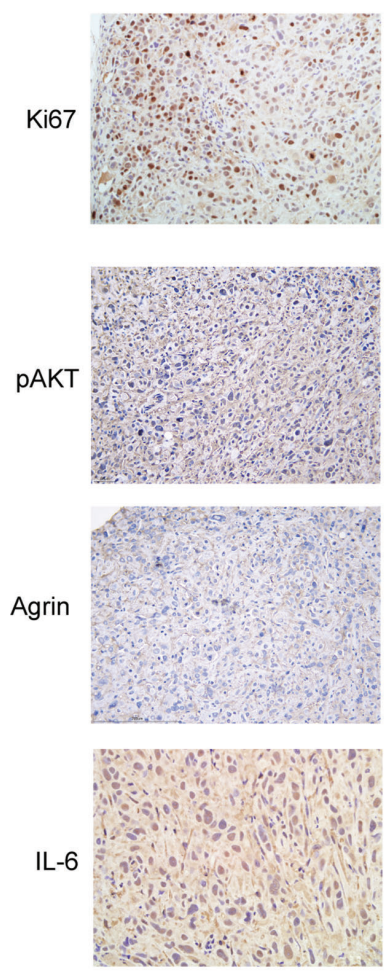

B

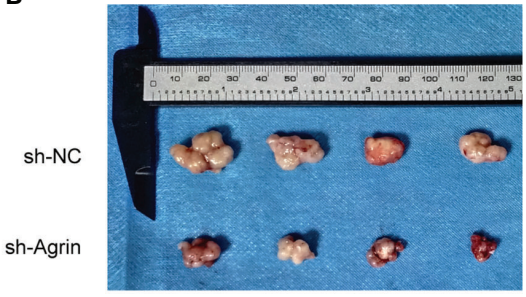

E

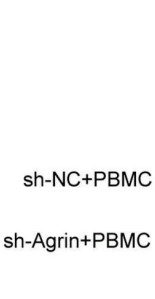

sh-Agrin
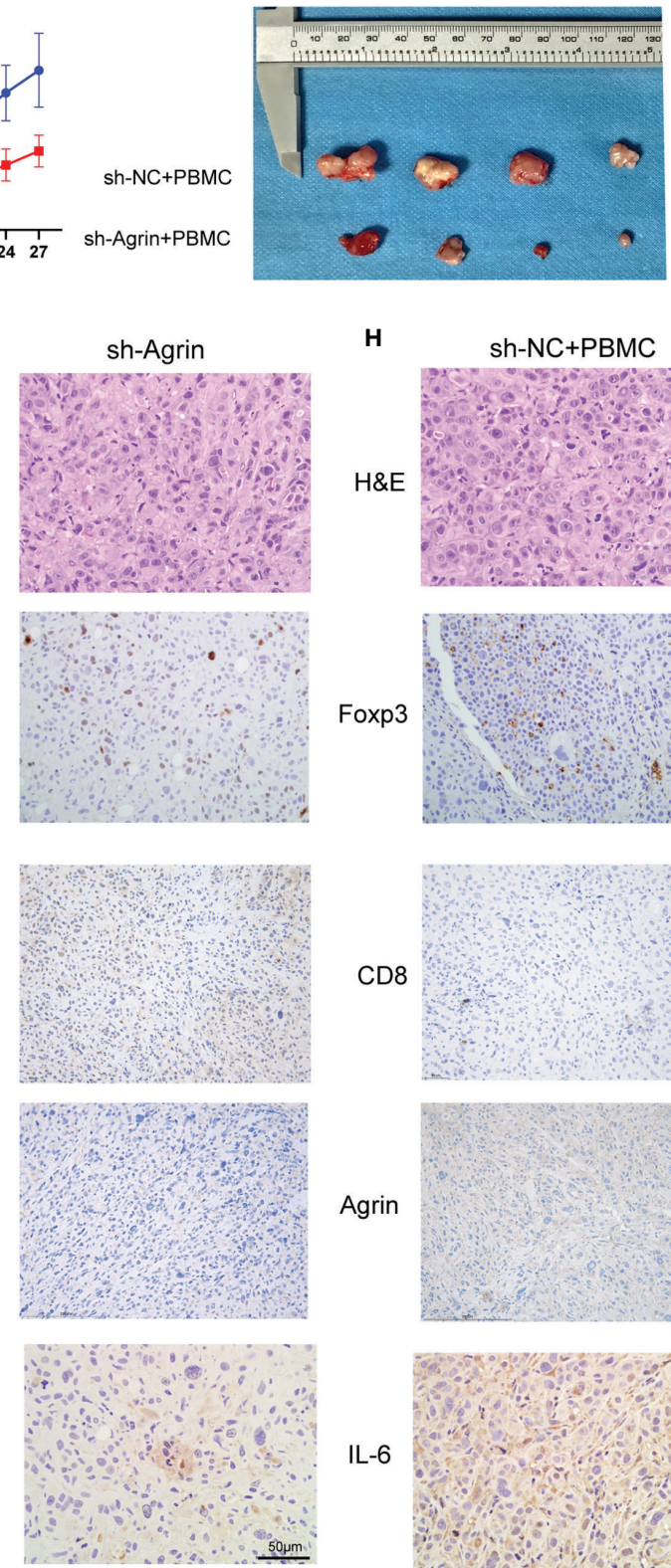

H

Foxp3

IL-6
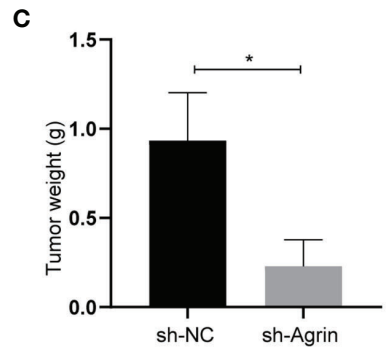

F
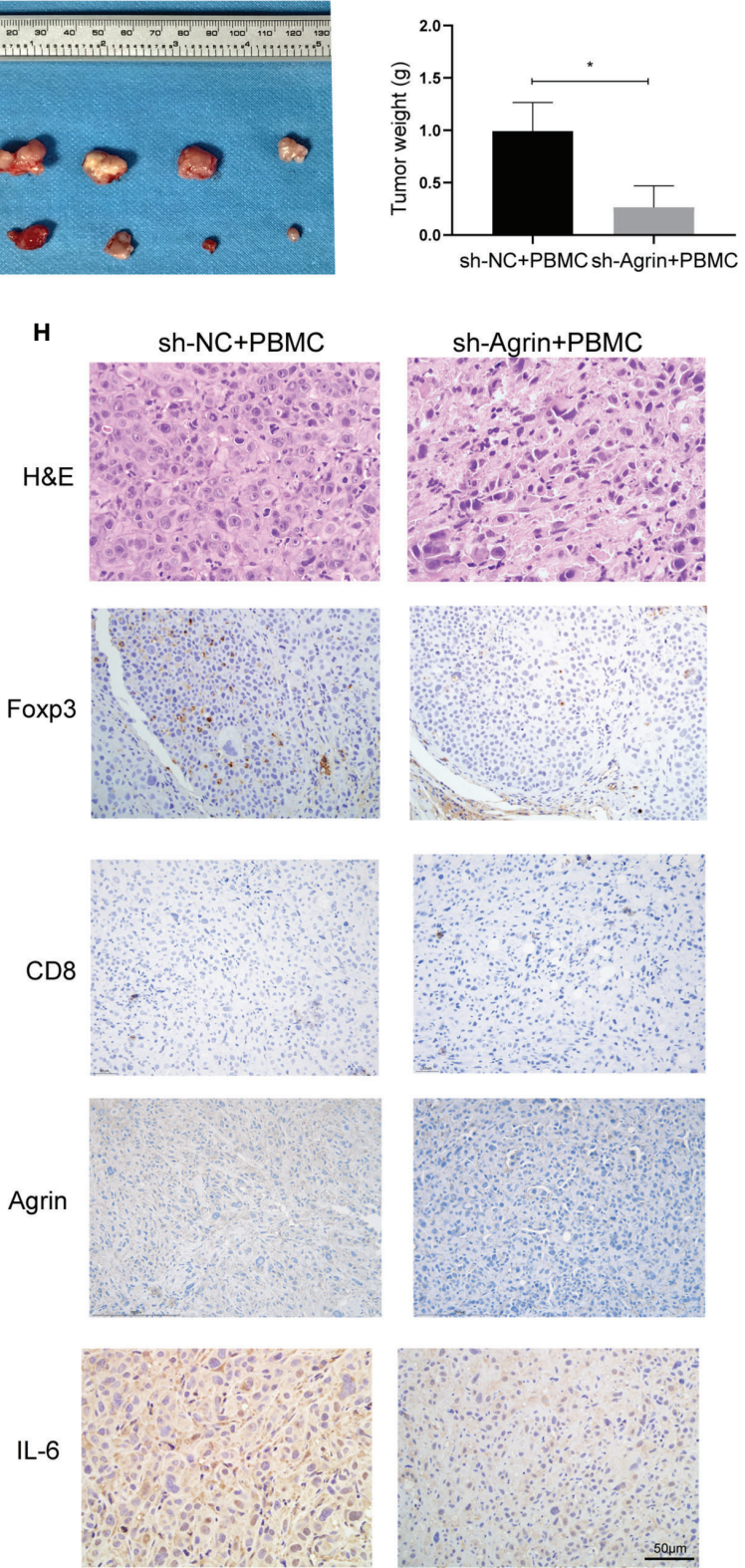

FIGURE 7 | Agrin silencing prohibited NSCLC cell growth, and alleviated IL-6, p-AKT and Foxp3 expression in vivo. (A-F) Agrin knockdown suppressed NSCLC cell growth in the xenograft tumor model of BALB/C nude mice with or without PBMC injection. (G, H) Representative images of Ki67, Foxp3, p-AKT, CD8, Agrin and IL6 staining in the tumor tissues. The images were taken at 200X magnification. Scale bar, $50 \mu \mathrm{m} . \mathrm{N} \geq 4$; ${ }^{*} \mathrm{P}<0.05$. 


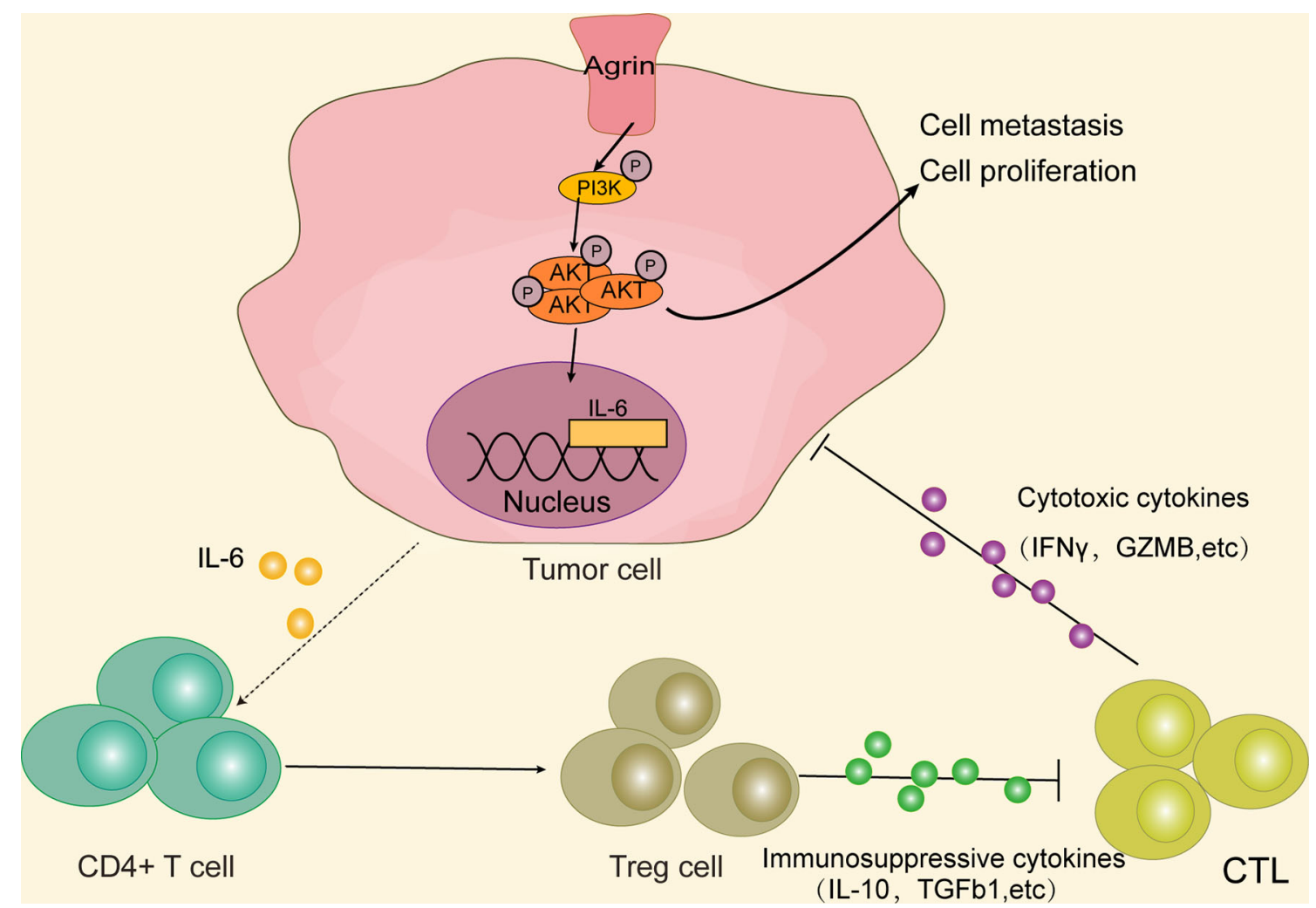

FIGURE 8 | Agrin promotes NSCLC progression and Treg differentiation via inducing PI3K/AKT signaling pathway. Agrin promotes NSCLC cell proliferation and metastasis, as well as IL-6 expression and secretion, via stimulating PI3K/AKT signaling pathway. IL-6 subsequently induces Treg differentiation. Immunosuppressive cytokines secreted by Tregs might inhibit cytotoxic T cells from releasing cytotoxic cytokines, ultimately triggering tumor immune evasion.

facilitate multiple tumor amplification via increasing cell viability (34, 35). Intracellular PI3K/AKT pathway is important for mediating epithelial-mesenchymal transition (EMT) via inhibiting E-cadherin transcription (36), inducing N-cadherin (37) and other key EMT drivers (such as Snail and Slug). Our enriched analysis showed that Agrin was correlated with PI3K/ pAKT signaling pathway. Following studies indicated that PI3K/ AKT activators mitigated the effects of Agrin downregulation on NSCLC cells. Previous studies have shown that agrin can phosphorylate and activate Src kinase (38). Src/PI3K/AKT axis plays important roles in the development and drug resistance of various tumors (39-44). In lung cancer, Src/Akt pathway can promote the invasion and metastasis of lung cancer cells, suggesting that Src kinase may be an important factor for Agrin in regulating $\mathrm{PI} 3 \mathrm{~K} / \mathrm{AKT}$ signal and causing tumor development $(41,42,45,46)$. In addition, Wang et al. reported that Agrin in rectal cancer induced WNT pathway to promote rectal cancer development (47). Our results also verified WNT pathway-related proteins (c-myc, GSK-3 $\beta$, $\beta$-cantein) were downregulated in the Agrin-deficient groups (Figure S2G). Glycogen synthase kinase-3 $\beta$ (GSK-3 $\beta$ ) is a key protein in WNT pathway, also known as a tumor inhibitor. AKT could phosphorylate and inactivate GSK-3 $\beta$ at Ser9 $(48,49)$, which subsequently stabilized Snail protein and finally caused EMT $(50,51)$. Consistently, accumulated evidence revealed that activation of PI3K/AKT/GSK-3 $\beta$ pathway resulted in lung cancer progression (52), hepatocellular carcinoma metastasis (53) and so on. These data suggested that Agrin might exert its prooncogenic function via PI3K/AKT pathway, with GSK-3 $\beta$ as a potential downstream target.

Based on the reports of Agrin in auto-immune diseases and T cells, we further explored the influence of Agrin on T cells in NSCLC TIME. Agrin was positively correlated with Treg infiltration and negatively associated with the response of immune checkpoint inhibitors in NSCLC patients. After coculture with NSCLC cells, the proportion of Tregs (CD4+, CD25+, Foxp3+) in PBMCs was decreased in the Agrin-deficient group. Tregs exert immunosuppressive mechanism in 2 ways: secret cytokines and direct contact (22). Treg-produced immunosuppressive cytokines (TGF-b1, IL-10) restrain the function of T effector cells (54-56). The cytokines secreted from Tregs, such as perforin and granzyme B, could directly kill anti-cancer immune cells, including antigen presenting cells and $\mathrm{T}$ effector cells. Moreover, immune checkpoint CTLA-4 on the surface of Tregs bind to their targets on anti-cancer immune cells, and inhibit their functions. A key transcription factor for Treg function is Foxp3, forced expression of which coverts naïve T cells into immunosuppressive Treg-like cells (57). These studies indicated that Treg-related cytokines and immune biomarker might be important for the immunosuppressive functions of Tregs. 
In the present work, Treg-related mRNAs were downregulated in Jurkat $\mathrm{T}$ cells and PBMCs after cocultured with Agrin-deficient NSCLC cells, suggesting that knocking down Agrin in NSCLC cells declined Treg differentiation in TIME. Based on the findings that PI3K/AKT pathway participated in Agrin-facilitating NSCLC progression, we assumed that PI3K/AKT pathway might be a pivotal factor for Agrin-promoting Treg differentiation. As expected, the decreased Treg differentiation was abrogated after the addition of PI3K/AKT activators in the Agrin-deficient NSCLC cells.

Various cytokines are involved in the process of Treg differentiation, including IL-6, IL-10 and TGF-b1 (58). Agrin downregulation suppressed IL-6 production, followed by the decrease of Treg differentiation. IL- 6 was reported to mediate various solid cancer behaviors, such as tumor growth and metastasis (59-62). Moreover, IL-6 is associated with poor prognosis of lung cancer patients (63). In addition, patients with elevated levels of circulating IL- 6 are more likely to have higher Treg proportions and resistance to treatments (64-66), implicating that blocking IL-6 might result in therapeutic gain of NSCLC therapy. However, little research was concentrated on its roles in TIME. In our study, we first demonstrated that IL-6 was a critical mediator for Agrin to facilitate Treg infiltration in NSCLC. Based on the tumor-promoting characteristic of PI3K/ AKT pathway and previous studies on AKT-regulating IL-6 expression, we subsequently assessed the effects of PI3K/AKT activators on IL-6 levels. From qPCR, immunoblotting and ELISA, we found that SC79 restored the expression and secretion of IL-6 in Agrin-deficient NSCLCs. These results implicated that PI3K/pAKT pathway was necessary for Agrinstimulated IL- 6 production.

Due to the lack of immune competence, our nude mouse model insufficiently simulated complex immune regulatory responses in tumor microenvironments in vivo. Further investigation on function of Agrin in immune-competent mice would be required in future studies.

\section{CONCLUSIONS}

In summary, our study originally indicated that higher Agrin levels were associated with worse survival in NSCLC patients. Agrin enhanced tumor growth and invasion through PI3K/AKT pathway, and induced IL-6 expression and secretion in NSCLC cells to promote Treg differentiation. Therefore, Agrin/PI3K/AKT/IL-6 axis might be a novel potential target for NSCLC therapies (Figure 8).

\section{REFERENCES}

1. Sung H, Ferlay J, Siegel RL, Laversanne M, Soerjomataram I, Jemal A, et al. Global Cancer Statistics 2020: GLOBOCAN Estimates of Incidence and Mortality Worldwide for 36 Cancers in 185 Countries. CA Cancer J Clin (2021) 71(3):209-49. doi: 10.3322/caac.21660

2. Bunn PA Jr, Dimou A. Systemic Therapy for Elderly Patients With Advanced Non-Small-Cell Lung Cancers. J Clin Oncol (2018) 36(25):2571-4. doi: 10.1200/JCO.2018.79.2457

3. Han L, Hongjie S, Yuan L, Wenjie S, Shuying L, Nannan Z, et al. Gene Signature Based on B Cell Predicts Clinical Outcome of Radiotherapy and

\section{DATA AVAILABILITY STATEMENT}

The original contributions presented in the study are included in the article/Supplementary Material. Further inquiries can be directed to the corresponding authors.

\section{ETHICS STATEMENT}

The studies involving human participants were reviewed and approved by The Institutional Review Board at Zhongnan Hospital of Wuhan University. The patients/participants provided their written informed consent to participate in this study. The animal study was reviewed and approved by The Institutional Animal Care and Use Committee at Zhongnan Hospital of Wuhan University.

\section{AUTHOR CONTRIBUTIONS}

LH, CX, and YGong designed the study. LH, HS, SM, YL, and WS acquired and interpretated the clinical and preclinical data. SL, NZ, XJ, YGao, and ZH collected and analyzed the in vitro and in vivo data. All authors read and approved the final manuscript.

\section{FUNDING}

This study was supported by National Natural Science Foundation of China (81800429, 81773236 and 81972852), Key Research \& Development Project of Hubei Province (2020BCA069), Nature Science Foundation of Hubei Province (2020CFB612), Health Commission of Hubei Province Medical Leading Talent Project, Young and Middle-Aged Medical Backbone Talents of Wuhan (WHQG201902), Application Foundation Frontier Project of Wuhan (2020020601012221), Zhongnan Hospital of Wuhan University Science, Technology and Innovation Seed Fund (znpy2019001 and znpy2019048), and Translational Medicine and Interdisciplinary Research Joint Fund of Zhongnan Hospital of Wuhan University (ZNJC201922 and ZNJC202007).

\section{SUPPLEMENTARY MATERIAL}

The Supplementary Material for this article can be found online at: https://www.frontiersin.org/articles/10.3389/fonc.2021.804418/ full\#supplementary-material

Immunotherapy for Patients With Lung Adenocarcinoma. Cancer Med (2020) 9(24):9581-94. doi: 10.1002/cam4.3561

4. Chen Z, Fillmore CM, Hammerman PS, Kim CF, Wong KK. Non-Small-Cell Lung Cancers: A Heterogeneous Set of Diseases. Nat Rev Cancer (2014) 14 (8):535-46. doi: 10.1038/nrc3775

5. Shi L, Fu AK, Ip NY. Molecular Mechanisms Underlying Maturation and Maintenance of the Vertebrate Neuromuscular Junction. Trends Neurosci (2012) 35(7):441-53. doi: 10.1016/j.tins.2012.04.005

6. Chakraborty S, Lakshmanan M, Swa HL, Chen J, Zhang X, Ong YS, et al. An Oncogenic Role of Agrin in Regulating Focal Adhesion Integrity in Hepatocellular Carcinoma. Nat Commun (2015) 6:6184. doi: 10.1038/ncomms7184 
7. Mundhenke C, Meyer K, Drew S, Friedl A. Heparan Sulfate Proteoglycans as Regulators of Fibroblast Growth Factor-2 Receptor Binding in Breast Carcinomas. Am J Pathol (2002) 160(1):185-94. doi: 10.1016/S0002-9440 (10)64362-3

8. Edwards IJ. Proteoglycans in Prostate Cancer. Nat Rev Urol (2012) 9(4):196206. doi: 10.1038/nrurol.2012.19

9. Kawahara R, Granato DC, Carnielli CM, Cervigne NK, Oliveria CE, Rivera C, et al. Agrin and Perlecan Mediate Tumorigenic Processes in Oral Squamous Cell Carcinoma. PloS One (2014) 9(12):e115004. doi: 10.1371/journal. pone. 0115004

10. Chakraborty S, Njah K, Pobbati AV, Lim YB, Raju A, Lakshmanan M, et al. Agrin as a Mechanotransduction Signal Regulating YAP Through the Hippo Pathway. Cell Rep (2017) 18(10):2464-79. doi: 10.1016/j.celrep.2017.02.041

11. Bromley SK, Burack WR, Johnson KG, Somersalo K, Sims TN, Sumen C, et al. The Immunological Synapse. Annu Rev Immunol (2001) 19:375-96. doi: 10.1146/annurev.immunol.19.1.375

12. Khan AA, Bose C, Yam LS, Soloski MJ, Rupp F. Physiological Regulation of the Immunological Synapse by Agrin. Science (2001) 292(5522):1681-6. doi: $10.1126 /$ science. 1056594

13. Jury EC, Eldridge J, Isenberg DA, Kabouridis PS. Agrin Signalling Contributes to Cell Activation and Is Overexpressed in T Lymphocytes From Lupus Patients. J Immunol (2007) 179(11):7975-83. doi: 10.4049/jimmunol. 179.11 .7975

14. Li D, Gu Q, Xie Z, Shen Q, Li H. Clinical Significance of Nuclear Localisation of Agrin in Lung Adenocarcinoma. Pol J Pathol (2019) 70(3):198-204. doi: 10.5114/pjp.2019.90396

15. Li T, Fan J, Wang B, Traugh N, Chen Q, Liu JS, et al. TIMER: A Web Server for Comprehensive Analysis of Tumor-Infiltrating Immune Cells. Cancer Res (2017) 77(21):e108-10. doi: 10.1158/0008-5472.CAN-17-0307

16. Fu J, Li K, Zhang W, Wan C, Zhang J, Jiang P, et al. Large-Scale Public Data Reuse to Model Immunotherapy Response and Resistance. Genome Med (2020) 12(1):21. doi: 10.1186/s13073-020-0721-z

17. Jiang P, Gu S, Pan D, Fu J, Sahu A, Hu X, et al. Signatures of T Cell Dysfunction and Exclusion Predict Cancer Immunotherapy Response. Nat Med (2018) 24(10):1550-8. doi: 10.1038/s41591-018-0136-1

18. Hanna N, Johnson D, Temin S, Baker S Jr, Brahmer J, Ellis PM, et al. Systemic Therapy for Stage IV Non-Small-Cell Lung Cancer: American Society of Clinical Oncology Clinical Practice Guideline Update. J Clin Oncol (2017) 35 (30):3484-515. doi: 10.1200/JCO.2017.74.6065

19. Zhou C, Yao LD. Strategies to Improve Outcomes of Patients With EGRFMutant Non-Small Cell Lung Cancer: Review of the Literature. J Thorac Oncol (2016) 11(2):174-86. doi: 10.1016/j.jtho.2015.10.002

20. Topalian SL, Drake CG, Pardoll DM. Immune Checkpoint Blockade: A Common Denominator Approach to Cancer Therapy. Cancer Cell (2015) 27(4):450-61. doi: 10.1016/j.ccell.2015.03.001

21. Huang M, Lopes GL, Insinga RP, Burke T, Ejzykowicz F, Zhang Y, et al. CostEffectiveness of Pembrolizumab Versus Chemotherapy as First-Line Treatment in PD-L1-Positive Advanced Non-Small-Cell Lung Cancer in the USA. Immunotherapy (2019) 11(17):1463-78. doi: 10.2217/imt-2019-0178

22. Ohue Y, Nishikawa H, Regulatory T. (Treg) Cells in Cancer: Can Treg Cells be a New Therapeutic Target? Cancer Sci (2019) 110(7):2080-9. doi: 10.1111/cas.14069

23. Wang H, Chen L. Tumor Microenviroment and Hepatocellular Carcinoma Metastasis. J Gastroenterol Hepatol (2013) 28(Suppl 1):43-8. doi: 10.1111/ jgh.12091

24. Roxburgh CS, Salmond JM, Horgan PG, Oien KA, McMillan DC. The Relationship Between the Local and Systemic Inflammatory Responses and Survival in Patients Undergoing Curative Surgery for Colon and Rectal Cancers. J Gastrointest Surg (2009) 13(11):2011-2018; discussion 20182019. doi: $10.1007 /$ s11605-009-1034-0

25. Hanahan D, Weinberg RA. Hallmarks of Cancer: The Next Generation. Cell (2011) 144(5):646-74. doi: 10.1016/j.cell.2011.02.013

26. Chen X, Song E. Turning Foes to Friends: Targeting Cancer-Associated Fibroblasts. Nat Rev Drug Discov (2019) 18(2):99-115. doi: 10.1038/s41573018-0004-1

27. Whiteside TL, Mandapathil M, Szczepanski M, Szajnik M. Mechanisms of Tumor Escape From the Immune System: Adenosine-Producing Treg, Exosomes and Tumor-Associated TLRs. Bull Cancer (2011) 98(2):E25-31. doi: $10.1684 /$ bdc.2010.1294
28. Kosmaczewska A, Ciszak L, Potoczek S, Frydecka I. The Significance of Treg Cells in Defective Tumor Immunity. Arch Immunol Ther Exp (Warsz) (2008) 56(3):181-91. doi: 10.1007/s00005-008-0018-1

29. Lu L, Barbi J, Pan F. The Regulation of Immune Tolerance by FOXP3. Nat Rev Immunol (2017) 17(11):703-17. doi: 10.1038/nri.2017.75

30. Fridman WH, Pages F, Sautes-Fridman C, Galon J. The Immune Contexture in Human Tumours: Impact on Clinical Outcome. Nat Rev Cancer (2012) 12 (4):298-306. doi: 10.1038/nrc3245

31. Onizuka S, Tawara I, Shimizu J, Sakaguchi S, Fujita T, Nakayama E. Tumor Rejection by In Vivo Administration of Anti-CD25 (Interleukin-2 Receptor Alpha) Monoclonal Antibody. Cancer Res (1999) 59(13):3128-33.

32. Kurose K, Ohue Y, Wada H, Iida S, Ishida T, Kojima T, et al. Phase Ia Study of FoxP3+ CD4 Treg Depletion by Infusion of a Humanized Anti-CCR4 Antibody, KW-0761, in Cancer Patients. Clin Cancer Res (2015) 21 (19):4327-36. doi: 10.1158/1078-0432.CCR-15-0357

33. Njah K, Chakraborty S, Qiu B, Arumugam S, Raju A, Pobbati AV, et al. A Role of Agrin in Maintaining the Stability of Vascular Endothelial Growth Factor Receptor-2 During Tumor Angiogenesis. Cell Rep (2019) 28(4):949-65.e947. doi: 10.1016/j.celrep.2019.06.036

34. Yuan TL, Cantley LC. PI3K Pathway Alterations in Cancer: Variations on a Theme. Oncogene (2008) 27(41):5497-510. doi: 10.1038/onc.2008.245

35. Graves EE, Maity A, Le QT. The Tumor Microenvironment in Non-SmallCell Lung Cancer. Semin Radiat Oncol (2010) 20(3):156-63. doi: 10.1016/ j.semradonc.2010.01.003

36. Larue L, Bellacosa A. Epithelial-Mesenchymal Transition in Development and Cancer: Role of Phosphatidylinositol 3' Kinase/AKT Pathways. Oncogene (2005) 24(50):7443-54. doi: 10.1038/sj.onc.1209091

37. Liang J, Li H, Han J, Jiang J, Wang J, Li Y, et al. Mex3a Interacts With LAMA2 to Promote Lung Adenocarcinoma Metastasis via PI3K/AKT Pathway. Cell Death Dis (2020) 11(8):614. doi: 10.1038/s41419-020-02858-3

38. Ramseger R, White R, Kroger S. Transmembrane Form Agrin-Induced Process Formation Requires Lipid Rafts and the Activation of Fyn and MAPK. J Biol Chem (2009) 284(12):7697-705. doi: 10.1074/jbc.M806719200

39. Nukaga S, Yasuda H, Tsuchihara K, Hamamoto J, Masuzawa K, Kawada I, et al. Amplification of EGFR Wild-Type Alleles in Non-Small Cell Lung Cancer Cells Confers Acquired Resistance to Mutation-Selective EGFR Tyrosine Kinase Inhibitors. Cancer Res (2017) 77(8):2078-89. doi: 10.1158/ 0008-5472.CAN-16-2359

40. Kanda R, Kawahara A, Watari K, Murakami Y, Sonoda K, Maeda M, et al. Erlotinib Resistance in Lung Cancer Cells Mediated by Integrin Beta1/Src/ Akt-Driven Bypass Signaling. Cancer Res (2013) 73(20):6243-53. doi: 10.1158/0008-5472.CAN-12-4502

41. Jiang W, Xu Z, Yu L, Che J, Zhang J, Yang J. MicroRNA-144-3p Suppressed TGF-Beta1-Induced Lung Cancer Cell Invasion and Adhesion by Regulating the Src-Akt-Erk Pathway. Cell Biol Int (2019) 44(1):51-61. doi: 10.1002/ cbin. 11158

42. Singh S, Trevino J, Bora-Singhal N, Coppola D, Haura E, Altiok S, et al. EGFR/ Src/Akt Signaling Modulates Sox2 Expression and Self-Renewal of Stem-Like Side-Population Cells in Non-Small Cell Lung Cancer. Mol Cancer (2012) 11:73. doi: $10.1186 / 1476-4598-11-73$

43. Lu Y, Yu Q, Liu JH, Zhang J, Wang H, Koul D, et al. Src Family ProteinTyrosine Kinases Alter the Function of PTEN to Regulate Phosphatidylinositol 3-Kinase/AKT Cascades. J Biol Chem (2003) 278 (41):40057-66. doi: 10.1074/jbc.M303621200

44. Zhao Y, Scott A, Zhang P, Hao Y, Feng X, Somasundaram S, et al. Regulation of Paxillin-P130-PI3K-AKT Signaling Axis by Src and PTPRT Impacts Colon Tumorigenesis. Oncotarget (2017) 8(30):48782-93. doi: 10.18632/ oncotarget.10654

45. Gong WJ, Liu JY, Yin JY, Cui JJ, Xiao D, Zhuo W, et al. Resistin Facilitates Metastasis of Lung Adenocarcinoma Through the TLR4/Src/EGFR/PI3K/NFkappaB Pathway. Cancer Sci (2018) 109(8):2391-400. doi: 10.1111/cas.13704

46. Chen B, Xu X, Luo J, Wang H, Zhou S. Rapamycin Enhances the Anti-Cancer Effect of Dasatinib by Suppressing Src/PI3K/mTOR Pathway in NSCLC Cells. PloS One (2015) 10(6):e0129663. doi: 10.1371/journal.pone.0129663

47. Wang ZQ, Sun XL, Wang YL, Miao YL. Agrin Promotes the Proliferation, Invasion and Migration of Rectal Cancer Cells via the WNT Signaling Pathway to Contribute to Rectal Cancer Progression. J Recept Signal Transduct Res (2021) 41(4):363-70. doi: 10.1080/10799893.2020.1811325 
48. McCubrey JA, Steelman LS, Bertrand FE, Davis NM, Sokolosky M, Abrams SL, et al. GSK-3 as Potential Target for Therapeutic Intervention in Cancer. Oncotarget (2014) 5(10):2881-911. doi: 10.18632/oncotarget.2037

49. Jin F, Wu Z, Hu X, Zhang J, Gao Z, Han X, et al. The PI3K/Akt/GSK-3beta/ ROS/eIF2B Pathway Promotes Breast Cancer Growth and Metastasis via Suppression of NK Cell Cytotoxicity and Tumor Cell Susceptibility. Cancer Biol Med (2019) 16(1):38-54. doi: 10.20892/j.issn.2095-3941.2018.0253

50. Zhou BP, Deng J, Xia W, Xu J, Li YM, Gunduz M, et al. Dual Regulation of Snail by GSK-3beta-Mediated Phosphorylation in Control of EpithelialMesenchymal Transition. Nat Cell Biol (2004) 6(10):931-40. doi: 10.1038/ ncb1173

51. Cohen P, Frame S. The Renaissance of GSK3. Nat Rev Mol Cell Biol (2001) 2 (10):769-76. doi: 10.1038/35096075

52. Chen L, Wu Q, Xu X, Yang C, You J, Chen F, et al. Cancer/testis Antigen LDHC Promotes Proliferation and Metastasis by Activating the PI3K/Akt/ GSK-3beta-Signaling Pathway and the in Lung Adenocarcinoma. Exp Cell Res (2021) 398(2):112414. doi: 10.1016/j.yexcr.2020.112414

53. Jiang $\mathrm{H}$, Zhou Z, Jin $\mathrm{S}$, Xu $\mathrm{K}$, Zhang $\mathrm{H}$, Xu J, et al. PRMT9 Promotes Hepatocellular Carcinoma Invasion and Metastasis via Activating PI3K/Akt/ GSK-3beta/Snail Signaling. Cancer Sci (2018) 109(5):1414-27. doi: 10.1111/ cas. 13598

54. Mahic M, Yaqub S, Johansson CC, Tasken K, Aandahl EM. FOXP3+CD4 $+\mathrm{CD} 25+$ Adaptive Regulatory T Cells Express Cyclooxygenase-2 and Suppress Effector T Cells by a Prostaglandin E2-Dependent Mechanism. J Immunol (2006) 177(1):246-54. doi: 10.4049/jimmunol.177.1.246

55. Baratelli F, Lin Y, Zhu L, Yang SC, Heuze-Vourc'h N, Zeng G, et al. Prostaglandin E2 Induces FOXP3 Gene Expression and T Regulatory Cell Function in Human CD4+ T Cells. J Immunol (2005) 175(3):1483-90. doi: 10.4049/jimmunol.175.3.1483

56. Yaqub S, Henjum K, Mahic M, Jahnsen FL, Aandahl EM, Bjornbeth BA, et al. Regulatory T Cells in Colorectal Cancer Patients Suppress Anti-Tumor Immune Activity in a COX-2 Dependent Manner. Cancer Immunol Immunother (2008) 57(6):813-21. doi: 10.1007/s00262-007-0417-x

57. Shimizu J, Yamazaki S, Takahashi T, Ishida Y, Sakaguchi S. Stimulation of CD25(+)CD4(+) Regulatory T Cells Through GITR Breaks Immunological Self-Tolerance. Nat Immunol (2002) 3(2):135-42. doi: 10.1038/ni759

58. Wang YA, Li XL, Mo YZ, Fan CM, Tang L, Xiong F, et al. Effects of Tumor Metabolic Microenvironment on Regulatory T Cells. Mol Cancer (2018) 17 (1):168. doi: 10.1186/s12943-018-0913-y

59. Sahin E, Baycu C, Koparal AT, Burukoglu Donmez D, Bektur E. Resveratrol Reduces IL-6 and VEGF Secretion From Co-Cultured A549 Lung Cancer
Cells and Adipose-Derived Mesenchymal Stem Cells. Tumour Biol (2016) 37 (6):7573-82. doi: 10.1007/s13277-015-4643-0

60. Sen M, Johnston PA, Pollock NI, DeGrave K, Joyce SC, Freilino ML, et al. Mechanism of Action of Selective Inhibitors of IL-6 Induced STAT3 Pathway in Head and Neck Cancer Cell Lines. J Chem Biol (2017) 10(3):129-41. doi: 10.1007/s12154-017-0169-9

61. Nguyen DP, Li J, Tewari AK. Inflammation and Prostate Cancer: The Role of Interleukin 6 (IL-6). BJU Int (2014) 113(6):986-92. doi: 10.1111/bju.12452

62. Bharti R, Dey G, Ojha PK, Rajput S, Jaganathan SK, Sen R, et al. DiacereinMediated Inhibition of IL-6/IL-6R Signaling Induces Apoptotic Effects on Breast Cancer. Oncogene (2016) 35(30):3965-75. doi: 10.1038/onc.2015.466

63. Silva EM, Mariano VS, Pastrez PRA, Pinto MC, Castro AG, Syrjanen KJ, et al. High Systemic IL-6 is Associated With Worse Prognosis in Patients With Non-Small Cell Lung Cancer. PloS One (2017) 12(7):e0181125. doi: 10.1371/ journal.pone. 0181125

64. Rice SJ, Liu X, Zhang J, Jia B, Zheng H, Belani CP. Advanced NSCLC Patients With High IL-6 Levels Have Altered Peripheral T Cell Population and Signaling. Lung Cancer (2019) 131:58-61. doi: 10.1016/j.lungcan.2019.03.014

65. Wu CT, Chen MF, Chen WC, Hsieh CC. The Role of IL-6 in the Radiation Response of Prostate Cancer. Radiat Oncol (2013) 8:159. doi: 10.1186/1748-717X-8-159

66. Chen MF, Chen PT, Lu MS, Lin PY, Chen WC, Lee KD. IL-6 Expression Predicts Treatment Response and Outcome in Squamous Cell Carcinoma of the Esophagus. Mol Cancer (2013) 12:26. doi: 10.1186/1476-4598-12-26

Conflict of Interest: The authors declare that the research was conducted in the absence of any commercial or financial relationships that could be construed as a potential conflict of interest.

Publisher's Note: All claims expressed in this article are solely those of the authors and do not necessarily represent those of their affiliated organizations, or those of the publisher, the editors and the reviewers. Any product that may be evaluated in this article, or claim that may be made by its manufacturer, is not guaranteed or endorsed by the publisher.

Copyright (C) 2022 Han, Shi, Ma, Luo, Sun, Li, Zhang, Jiang, Gao, Huang, Xie and Gong. This is an open-access article distributed under the terms of the Creative Commons Attribution License (CC BY). The use, distribution or reproduction in other forums is permitted, provided the original author(s) and the copyright owner(s) are credited and that the original publication in this journal is cited, in accordance with accepted academic practice. No use, distribution or reproduction is permitted which does not comply with these terms. 